\title{
La curva de rendimiento y su relación con la actividad económica en México
}

\author{
The yield curve and its relation to economic activity in Mexico \\ Martha Beatriz Mota Aragón ${ }^{\mathrm{a}}$ y Leovardo Mata Mata ${ }^{\mathrm{b} *}$ \\ ${ }^{a}$ Universidad Autónoma Metropolitana, México \\ ${ }^{b}$ Universidad Anáhuac, México
}

Recibido el 29 de agosto del 2016; aceptado el 05 de octubre del 2017

Disponible en Internet el 17 de septiembre de 2018

\section{Resumen}

En este trabajo se estudian los componentes principales de la variación de las tasas de interés de los instrumentos de deuda, emitidos por el Gobierno mexicano, en el periodo 1978-2017. Mediante un vector autorregresivo se encuentra una relación entre dichos componentes y las variables: brecha del PIB, tasa de inflación y tasa de crecimiento económico. Asimismo, se encuentra evidencia de causalidad en el sentido de Granger, lo que establece un puente entre la curva de rendimiento y la actividad económica en México.

Códigos JEL: G19, C51, C52.

Palabras clave: Curva de rendimiento; renta fija; componentes principales; actividad económica; causalidad en el sentido de Granger.

\footnotetext{
Abstract

In this paper are analyzed the main components of the interest rate variation of debt instruments, issued by the Mexican Government, in the period 1978-2017. By means of an autoregressive vector, there is

*Autor para correspondencia.

Correo electrónico: leovardomata@gmail.com (L. Mata)

La revisión por pares es responsabilidad de la Universidad Nacional Autónoma de México. 
a relationship between these components and the variables: GDP gap, inflation rate and economic growth rate. Likewise, there is evidence of causality in the Granger sense, which establishes a bridge between the yield curve and economic activity in Mexico.

JEL Classification: G19, C51, C52.

Keywords: Accruals: Yield curve; fixed income; principal components; economic activity; Granger causality.

\section{Introducción}

El análisis de las tasas de interés de los instrumentos de deuda en México es un ejercicio relevante, pues son una herramienta de política monetaria para el banco central, en tanto que los hogares y las personas basan sus decisiones en el nivel y cambio de las tasas de interés. En ese sentido, este documento busca contribuir al estudio de la estructura temporal de las tasas de interés ${ }^{1}$ para el caso mexicano, ya que el mercado de renta fija se ha tornado cada vez más sofisticado, debido en parte a la dinámica con los mercados internacionales de capital así como por la aparición de nuevos instrumentos financieros y la expansión-recesión de la economía, tal es el caso de la crisis financiera de 2008-2009.

En este trabajo se analizan los componentes principales de la variación de las tasas de interés de los instrumentos de deuda, emitidos por el Gobierno mexicano, en el periodo 1978-2017. Luego, mediante pruebas de causalidad en el sentido de Granger y un vector autorregresivo se encuentra una relación entre dichos componentes y las variables: brecha del PIB, tasa de inflación y tasa de crecimiento económico. Estos resultados permiten establecer un puente entre la curva de rendimiento y la actividad económica en México.

El documento se organiza como sigue: primero se revisa la literatura reciente sobre este tema, en la segunda sección se revisa la técnica de componentes principales y pruebas de hipótesis, en la tercera sección se presentan los resultados de un vector autorregresivo y finalmente las conclusiones.

\section{Revisión de literatura}

Un tema de gran interés es entender como el segmento corto de la estructura temporal de las tasas de interés se relaciona con las tasas de largo plazo, y que factores subyacentes puedan estar afectando tanto los niveles como las variaciones de las tasas, y como estos cambios se relacionan con el sector real de la economía. Bajo este contexto, las técnicas de componentes principales y análisis factorial han sido las herramientas utilizadas, Martínez y Núñez (2012) y García (2011).

Concretamente, como se desarrolla en Litterman y Scheinkman (1991) y posteriormente en Knez, Litterman y Scheinkman (1996), bajo la técnica de componentes principales se determinan los factores comunes que afectan los rendimientos sobre instrumentos de renta fija de los Estados Unidos. Para explicar la varianza de los rendimientos, es necesario distinguir o diferenciar el riesgo sistemático del riesgo particular que afecta a cada uno de los instrumentos. Los factores comunes con los que se busca explicar lo anterior son el nivel, la pendiente y la curvatura; sin embargo dependiendo del mercado al que se aplique la técnica

\footnotetext{
${ }^{1}$ Este término hace alusión a la relación existente (curva de rendimiento), en un momento dado del tiempo, entre el rendimiento de uno o más bonos y el tiempo que resta hasta su vencimiento, es decir se compara el rendimiento de diferentes bonos con diversos vencimientos en un instante del tiempo (Martínez y Núñez, 2012).
} 
de componentes principales y el momento histórico, no necesariamente son tres los factores, sino por ejemplo dos o uno. Como se menciona en Litterman y Scheinkman (1991) el enfoque tiene consecuencias sobre la posible cobertura que los agentes financieros pueden lograr al considerar el efecto sobre sus portafolios. Como se menciona en Jiménez (2002) otra posible aplicación del análisis de componentes principales se ubica en los modelos de estructuras de tasas, para encontrar los parámetros de las funciones de volatilidad.

Entre los trabajos a nivel internacional se encuentra el de Bühler y Zimmerman (1996) quienes estudian los casos de Alemania y Suiza para los cambios de tasas de interés, usando la matriz de correlaciones. Los autores destacan la complejidad con la que se puede explicar la evolución de los cambios en las tasas debido a diversos factores como el cambio en el ambiente institucional. Además la creciente incertidumbre de las tasas de interés en el periodo de estudio resaltó la importancia y uso de diversos instrumentos derivados como los swaps y las opciones; de esta manera ya se vislumbraba lo que hoy es muy común: la cobertura del riesgo de tasa de interés no es trivial. La relevancia del trabajo Litterman y Scheinkman (1991) es clara puesto que plantean que la complicada estructura de tasas puede ser caracterizada por un pequeño número de factores comunes (cuando mucho tres) y que afectan los rendimientos de los bonos. En particular el trabajo, de Bühler y Zimmerman (1996) encuentra que el primer factor no refleja un movimiento paralelo de la estructura de plazos y que la correlación entre las tasas de interés decrece con el aumento del horizonte de tiempo.

Otra variante se encuentra en D'Ecclesia y Zenios (1994), donde se realiza un estudio con datos semanales para el mercado italiano, que en ese momento se encontraba con problemas de inflación, y por tanto la mayor parte de los instrumentos eran de corto plazo y tasa flotante; debido a eso a mediados de los ochenta el gobierno italiano reorganizó el mercado de sus bonos. Los datos usados abarcaron el periodo de 1988 a 1992 y se tenía como objetivo revisar si los rendimientos de diferentes plazos pueden explicarse por los cambios en un número pequeño de factores, bajo la técnica de componentes principales. En efecto, con tres componentes se explicaba el $99.82 \%$. En particular el primer factor explicaba el $93.91 \%$ de los cambios y puede interpretarse como un movimiento paralelo de la curva de rendimientos (nivel). Análogamente, el segundo y tercer factores se podían interpretar como la pendiente y la curvatura de la curva de rendimiento. Además se había encontrado evidencia de que la inmunización bajo estos tres factores es mejor que la medida de duración.

De manera similar, Barber y Cooper (1996) empleó un análisis por componentes principales sobre datos históricos de tasas de interés spot para determinar un conjunto de patrones fundamentales que permitieran anticipar cambios en dicha tasa, partiendo de la matriz de varianzas y covarianzas. Adicionalmente encontró cómo se puede utilizar esa información con fines de inmunización. Este documento se distingue de Litterman y Scheinkman (1991) en que se aplica la técnica de componentes principales sobre las variaciones de las tasas más que sobre los niveles, además de que se plantea como inmunizar a una cartera en el sentido tradicional de Redington (1952).

En un trabajo para México, Jiménez (2002), además de usar la técnicas de componentes principales y estudiar si se usa o no el mismo número de componentes, se estudia si los porcentajes de variación explicados por cada uno de ellos es constante o no. El periodo de análisis fue 1996-1999, el cual se dividió en cuatro etapas, de acuerdo a eventos nacionales e internacionales. El autor concluye que en efecto hay una variación importante en el porcentaje explicado por cada una de las tres componentes, lo cual tiene consecuencias en la estrategia 
de cobertura que los agentes financieros puedan llevar a cabo.

En otra investigación aplicada para México de Cortés, Ramos y Torres (2009), se encuentra que la curva de rendimiento es tal que el porcentaje de variación explicado por la primera y segunda componente es de $95.01 \%$, y las tres primeras componentes el $99.31 \%$. Su estudio también relaciona las componentes con variables macroeconómicas como medidas de inflación.

En otro trabajo aplicado, Martínez y Núñez (2012) encuentra también dos componentes como importantes en la explicación de la variación de los rendimientos, a saber, el primer componente (nivel) explica el $68.2 \%$ y el segundo componente (pendiente) el $27.6 \%$, que puede ser considerada como un proxy de la diferencia entre las tasas de menor y mayor plazo; esta idea original parte del trabajo de Ang y Piazzesi (2003).

Finalmente, cabe resaltar que diversas estimaciones sobre los diferenciales de las tasas de interés y el IGAE en Cerecero, Salazar y Salgado (2008) muestran evidencia indirecta de que la pendiente de la curva de rendimiento es un predictor asimétrico de la actividad económica en el periodo 2001-2008. De igual manera, Castellanos y Camero (2003) encuentran en el periodo 2002-2011 que la pendiente de la curva de rendimiento se relaciona con algunas variables macroeconómicas. En este trabajo se estudia el periodo completo 1978-2017 de las tasas de interés de los instrumentos de deuda del Gobierno mexicano y se busca evidencia sobre su relación con la tasa de inflación, la brecha del PIB y la tasa de crecimiento económico bajo componentes principales, causalidad en el sentido de Granger y un vector autorregresivo. Por ello, en la siguiente sección se presenta el procedimiento y técnicas que se usarán más adelante.

\section{Procedimientos y herramientas utilizadas}

La técnica de componentes principales (ACP) es un método que permite reducir un conjunto de variables explicativas en un conjunto más pequeño de variables, llamados componentes principales, que resumen en gran medida la información contenida en las variables originales. De esta forma se reduce la dimensión del conjunto inicial de información, Johnson y Wichern (2000).

Los componentes principales se pueden interpretar como variables latentes no observables que explican la máxima variación de los datos. Formalmente, cada variable latente se puede definir como una combinación lineal de las variables originales.

Específicamente, ACP encuentra transformaciones ortogonales de las variables explicativas y permite delinear una matriz de datos al reducir el número de variables analizadas. Mediante ACP se espera que solo unas pocas variables latentes expliquen la mayor parte de la variabilidad, así se reduce la dimensionalidad y se simplifica el problema en estudio.

Si se tienen $\mathrm{p}$ variables aleatorias observadas $X_{1}, X_{2}, \ldots, X_{p}$, se encuentran geométricamente unos ejes nuevos que señalan la dirección de máxima variabilidad. De hecho, si las variables son altamente colineales y presentan información común, entonces la dimensión real de los datos es menor que p. Usualmente se emplea la matriz de correlaciones de las variables explicativas para evitar problemas de escala o unidades de medida (Cuadras, 2014).

Si se denota con $X_{1}, X_{2}, \ldots, X_{p}$ las variables observables y con $Y_{1}, Y_{2}, \ldots, Y_{p}$ las componentes principales, entonces se puede emplear notación matricial para simplificar los cálculos, así $X$ denotará la matriz de variables originales y la matriz Y los componentes principales. 
Se buscan combinaciones lineales de la forma

$$
\begin{gathered}
Y_{1}=a_{11} X_{1}+a_{12} X_{2}+\cdots+a_{1 p} X_{p} \\
Y_{2}=a_{21} X_{1}+a_{22} X_{2}+\cdots+a_{2 p} X_{p} \\
Y_{p}=a_{p 1} X_{1}+a_{p 2} X_{2}+\cdots+a_{p p} X_{p} \\
Y=X A
\end{gathered}
$$

donde $Y$ es la matriz que contiene las puntuaciones de cada uno de las observaciones sobre las componentes y $A$ es una matriz que contiene los coeficientes de las combinaciones lineales. Por convención, $Y_{1}$ será aquella componente que explique la mayor parte de la variabilidad, $Y_{2}$ será ortogonal a $Y_{1}$ y explicará la mayor parte de la variabilidad restante y similarmente para el resto de los componentes. Los componentes principales son no correlacionadas y

$$
V\left[Y_{1}\right] \geq V\left[Y_{2}\right] \geq \cdots \geq V\left[Y_{p}\right] \geq 0
$$

El porcentaje de la varianza contenida por la i-ésima componente principal viene dado por $\lambda_{i} / \sum_{i=1}^{p} \lambda_{i}$, siendo que cada $\lambda_{i}$ es un valor propio de la matriz de correlaciones asociada a la matriz X.

Existen diferentes procedimientos para discernir el número representativo de componentes principales:

a) El criterio de Káiser es un procedimiento muy popular y consiste en retener aquellos componentes cuyos valores propios son superiores a la unidad, Johnson y Wichern (2000).

b) El método gráfico, en el cual la magnitud de los valores propios se grafica en orden descendente, sugiere quedarse con los componentes de los valores propios hasta que se observe el decremento más pronunciado.

c) Otra forma es conservar todos los componentes necesarios para acumular al menos entre el $80 \%$ y el $90 \%$ de la variación de los datos.

d) Un procedimiento más sofisticado consiste en simulación paralela, donde se conservan los componentes principales cuya magnitud es estadísticamente significativa y diferente de un ruido blanco, Dinno (2009).

En algunas ocasiones no es posible encontrar una interpretación directa para los componentes principales, así que se recomienda aplicar una rotación ortogonal, la cual busca minimizar el número de variables con saturaciones altas y encontrar un conjunto de componentes principales más sencilla de interpretar (rotación Varimax).

En cualquier caso, bajo la especificación de los componentes principales encontrados es posible analizar el conjunto de datos original y llegar a conclusiones que no se tenían en un primer momento.

Asimismo, mediante un enfoque asintótico según la normal multivariante para la matriz de datos $X$, se pueden construir intervalos de confianza tanto para los valores propios $\lambda_{i}$ 
como para los coeficientes $a_{i j}$ que determinan los componentes principales (Cuadras, 2014). Específicamente el intervalo de $(1-\vec{a}) \times 100 \%$ de confianza para los valores propios son

$$
\frac{\hat{\lambda}_{i}}{\sqrt{1+a z_{\alpha / 2}}}<\lambda_{i}<\frac{\hat{\lambda}_{i}}{\sqrt{1-a z_{\alpha / 2}}}
$$

donde $a^{2}=2 /(n-1)$ y $P\left[|Z|>Z_{\frac{\alpha}{2}}\right]=\alpha / 2, Z \sim N(0,1)$. En el caso de los valores propios $\hat{u}=\left[\hat{u}_{1}, \ldots, \hat{u}_{p}\right]$ se cumple que $\hat{u}_{i} \sim N_{p}\left(u_{i}, V_{i} / n\right)$ siendo

$$
V_{i}=\lambda_{i} \sum_{j \neq i} \frac{\lambda_{i}}{\left(\lambda_{i}-\lambda_{j}\right)^{2}} u_{i} u_{i}^{\prime}
$$

así que mediante los estimadores $\hat{\mu}_{i}$ y $\hat{V}_{i}$ se puede construir asintóticamente un intervalo de confianza (Cuadras, 2014).

Adicionalmente a este proceso de inferencia debe verificarse si las variables originales se encuentran altamente correlacionadas y se justifique la implementación de ACP. Existen dos pruebas de hipótesis clásicas en este respecto: la prueba de esfericidad de Barlett (EB) y el indicador Kaiser-Meyer-Ollin (KMO). La hipótesis nula en EB señala que la matriz de correlaciones R es igual a la matriz identidad, Johnson y Wichern (2000). El estadístico de prueba - $\ln (\operatorname{det}(R))$ sigue una distribución ji-cuadrada con $p(p-1) / 2$ grados de libertad. Por otra parte el estadístico KMO es un índice entre cero y uno, donde el valor superior a 0.70 señala una adecuación muestral aceptable. Un procedimiento numérico para evaluar los componentes principales y su sentido estadístico, consiste en emplear matrices aleatorias y comparar los resultados con los datos observados, de tal suerte que, si existe diferencia significativa, se puede afirmar que el componente principal captura información relevante (Dinno, 2009).

Una vez encontrados los componentes principales de las primeras diferencias de las tasas de interés, se estimará un vector autorregresivo (VAR) para indagar el impacto de los componentes principales sobre la tasa de inflación, la brecha del PIB y la tasa de crecimiento económico, en periodicidad trimestral y tomando en cuenta tres ventanas de tiempo de crecimiento estacionario.

Concretamente, la especificación es

$$
y_{t}=\pi_{0}+\pi_{1} y_{t-1}+\pi_{2} y_{t-2}+\cdots+\pi_{q} y_{t-q}+e_{t}
$$

donde $y_{t}$ es un vector de variables endógenas que incluye la tasa de inflación, la brecha del PIB, la tasa de crecimiento económico y los componentes principales del periodo ${ }^{2}$, q es el número de rezagos óptimos según los criterios de información de Akaike y Schwarz (Greene, 2010), $\pi_{\mathrm{j}}$ es una matriz de coeficientes y $e_{t}$ es un vector de perturbaciones aleatorias. El objetivo es encontrar evidencia de causalidad en el sentido de Granger y medir el impacto que tienen los componentes principales, que podrían interpretarse como el nivel y pendiente de la curva de rendimiento (Litterman y Scheinkman (1991)), en las variables: tasa de inflación,

\footnotetext{
${ }^{2}$ En este modelo se verifica que las series de tiempo correspondientes sean estacionarias y que presentan causalidad en el sentido de Granger (Enders, 2004).
} 
brecha del PIB y tasa de crecimiento económico. La siguiente sección desarrolla el conjunto de estimaciones y presenta los resultados encontrados.

\section{Estimaciones y resultados}

Noriega y Rodríguez-Peréz (2011) encuentra que la evolución del nivel del producto real de México entre 1895 y 2008 se puede presentar adecuadamente mediante un modelo estacionario en tendencia. La especificación que emplea identifica cuatro cambios estructurales, cuya ocurrencia coincide con cambios institucionales domésticos, guerras y crisis económicofinancieras. Concretamente existen cuatro etapas de crecimiento estacionario: 1895-1924, 1935-1952, 1956-1978 y 1989-2008, separados por tres épocas de transición: 1925-1934, 1953-1955 y 1979-1988.

Por ello, el conjunto de estimaciones que se realizarán conforme a la tasa de crecimiento económico (PIB), la tasa de inflación (INFLACIÓN) y la brecha del PIB (BRECHA) tomarán en cuenta las ventanas de tiempo ${ }^{3}$ : 1978-1988, 1989-2008 y 2009-2016, véase figura 1.

En la Tabla 1 se presentan las pruebas de hipótesis para verificar si las series de tiempo de las tasas de interés, en niveles y primera diferencia, son estacionarias. Las pruebas se realizan también para la brecha del PIB, la tasa de inflación y la tasa de crecimiento económico.

Se puede observar que bajo la prueba Kwiatkowski-Phillips-Schmidt-Shin (KPSS) no se rechaza la hipótesis de estacionariedad y que bajo los tests de Dickey-Fuller Aumentada (DFA) y Phillips-Perron (PP) se confirma la no estacionariedad en niveles.

Los resultados de la tabla 1 siguen las observaciones presentadas por Lardic, Priaulet y Priaulet (2003), quien argumenta en favor de utilizar la primera diferencia de las tasas de interés para realizar análisis multivariante del tipo factorial o componentes principales. En este sentido, se realizan las estimaciones de componentes principales empleando la matriz de correlaciones de los cambios de las tasas de interés para los tres subperiodos 1978-1988, 1989-2008 y 2009-2016. Se utiliza la matriz de correlación, ya que permite reducir el sesgo de estimación atribuible a las diferencias heterogéneas que existen en las tasas de interés observadas según sus plazos, Lardic, Priaulet y Priaulet (2003) y Martínez y Núñez (2012).

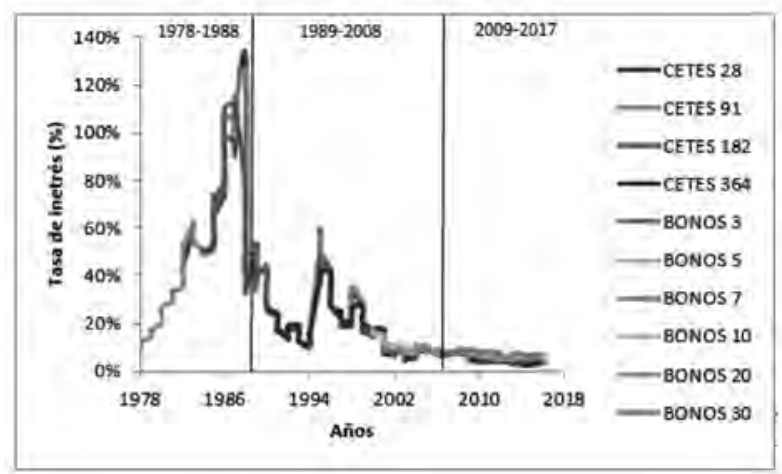

Figura 1 Tasas de interés anualizadas 1978-2017.

Fuente: elaboración propia con datos de Banxico.

\footnotetext{
${ }^{3}$ Los CETES son instrumentos que se emitieron por primera vez en 1978, por lo que se está considerando el periodo completo de instrumentos de deuda emitidos por el Gobierno que registra el caso mexicano.
} 
Para evaluar la pertinencia de la estimación por componentes principales se emplea el criterio de Kaiser-Meyer-Ollin (KMO) y la prueba de esfericidad de Barlett (EB). En este caso se presenta un nivel de adecuación muestral regular para la primera diferencia de las series de tiempo, ya que KMO se ubica ligeramente por arriba de 0.70. Además, se rechaza la hipótesis nula EB y se puede afirmar que las variables se encuentran altamente correlacionadas, ver Tabla 2.

Asimismo, se realiza la prueba de Doornik-Hansen para verificar si las variables se comportan como una normal multivariante (Mardia, Kent y Bibby, 1979) y se encuentra que sólo para el periodo 2009-2016 no se rechaza la hipótesis nula. En esta situación, se realizan cálculos e inferencias bajo el enfoque asintótico señalado por (Cuadras, 2014) para el periodo completo, 1978-2017.

Tabla 1

Pruebas de estacionariedad 1978-2017.

\begin{tabular}{|c|c|c|c|c|c|c|}
\hline \multirow{3}{*}{ Variable } & & & \multicolumn{2}{|c|}{ Estadísticos de prueba } & & \\
\hline & \multicolumn{2}{|r|}{ DFA } & \multicolumn{2}{|r|}{ PP } & \multicolumn{2}{|r|}{ KPSS } \\
\hline & Nivel & Diferencia & Nivel & Diferencia & Nivel & Diferencia \\
\hline CETES28 & -2.04 & $-10.93^{* * *}$ & -1.97 & $-13.08 * * *$ & $1.05 * * *$ & 0.06 \\
\hline CETES91 & -1.59 & $-10.76^{* * *}$ & -1.95 & $-11.18^{* * *}$ & $1.04 * * *$ & 0.05 \\
\hline CETES182 & -2.25 & $-5.45 * * *$ & -1.78 & $-7.93 * * *$ & $0.91 * * *$ & 0.04 \\
\hline CETES364 & -1.74 & $-9.40 * * *$ & -1.92 & $-9.44 * * *$ & $0.89 * * *$ & 0.04 \\
\hline BONOS3 & $-2.71 *$ & $-8.18 * * *$ & $-3.01 * *$ & $-8.19 * * *$ & $0.88 * * *$ & 0.29 \\
\hline BONOS5 & $-3.52 * *$ & $-6.84 * * *$ & $-3.89 * * *$ & $-6.83 * * *$ & $0.88 * * *$ & 0.48 \\
\hline BONOS7 & -2.34 & $-8.06^{* * *}$ & -2.30 & $-9.20 * * *$ & $0.89 * * *$ & 0.21 \\
\hline BONOS10 & -1.84 & $-9.23 * * *$ & -1.73 & $-9.75 * * *$ & $0.88 * * *$ & 0.12 \\
\hline BONOS20 & -2.06 & $-8.42 * * *$ & -1.96 & $-9.99 * * *$ & $0.75 * * *$ & 0.11 \\
\hline \multirow[t]{2}{*}{ BONOS30 } & -1.88 & $-7.03 * * *$ & -1.92 & $-7.03 * * *$ & $0.58 * * *$ & 0.06 \\
\hline & Nivel & Tasa de variación & Nivel & Tasa de variación & Nivel & Tasa de variación \\
\hline PIB & -3.63 & $-16.95 * * *$ & -3.92 & $-16.52 * * *$ & $1.06^{* * *}$ & 0.22 \\
\hline INFLACION & -3.84 & $-3.07 * *$ & -3.69 & $--2.94 * * *$ & $0.94 * *$ & 0.15 \\
\hline BRECHA & -4.18 & $-3.311^{*}$ & -3.88 & $-3.24 * *$ & $0.88 * *$ & 0.12 \\
\hline
\end{tabular}

Fuente: elaboración propia con datos de Banxico.

***: nivel de significancia al $10 \%$

***: nivel de significancia al $5 \%$

***: nivel de significancia al $1 \%$

En el Tabla 3 se muestran los valores propios de los dos componentes principales que se han retenido, de acuerdo al criterio de simulación paralela (SP) (Dinno, 2009), ver anexos. Se puede señalar que los componentes elegidos coinciden con los valores propios mayores a la unidad, según sugiere (Johnson y Wichern, 2000). La variación acumulada bajo los componentes es superior a $80 \%$ en cada uno de los subperiodos considerados. En el primer tramo, 1978-1988, sólo el primer componente es significativo, según SP, además de que sólo el primer componente cuenta con un valor propio mayor a la unidad.

En el Tabla 4 se muestran los componentes principales retenidos para cada uno de los subperiodos, junto con sus intervalos de confianza al 95\%. En el tramo 1978-1988 sólo es relevante el primer componente principal, lo que sugiere que en ese periodo la parte significativa de la curva de rendimiento sólo era su nivel. 
En cambio para los dos subperiodos siguientes, tanto el nivel como la pendiente de la curva se vuelven relevantes, ya que sus valores propios son mayores a la unidad y bajo SP tienen significado estadístico.

Tabla 2

Adecuación muestral para aplicar ACP.

\begin{tabular}{llll} 
& $1978-1988$ & $1989-2008$ & $2009-2017$ \\
\hline KMO & 0.7103 & 0.7120 & 0.7302 \\
EB & $57.032 * * *$ & $2238.513 * * *$ & $630.891 * * *$ \\
Normal multivariante & $56.616^{* * * *}$ & $611.881 * * *$ & 22.388 \\
\hline
\end{tabular}

Fuente: elaboración propia con datos de Banxico.

***: nivel de significancia al $10 \%$

***: nivel de significancia al $5 \%$

***: nivel de significancia al $1 \%$

Tabla 3

Valores propios bajo ACP.

\begin{tabular}{|c|c|c|c|c|c|c|}
\hline & \multicolumn{2}{|c|}{ 1978-1988 } & \multicolumn{2}{|c|}{ 1989-2008 } & \multicolumn{2}{|c|}{ 2009-2017 } \\
\hline & $\begin{array}{l}\text { Valores } \\
\text { propios }\end{array}$ & $\begin{array}{l}\text { Variación } \\
\text { acumulada }\end{array}$ & $\begin{array}{l}\text { Valores } \\
\text { propios }\end{array}$ & $\begin{array}{l}\text { Variación } \\
\text { acumulada }\end{array}$ & $\begin{array}{l}\text { Valores } \\
\text { propios }\end{array}$ & $\begin{array}{l}\text { Variación } \\
\text { acumulada }\end{array}$ \\
\hline Componente 1 & $\begin{array}{c}2.3561 \\
(1.90,3.49)\end{array}$ & $78.540 \%$ & $\begin{array}{c}5.2713 \\
(5.60,6.36)\end{array}$ & $52.710 \%$ & $\begin{array}{c}5.5416 \\
(4.50,7.96)\end{array}$ & $55.429 \%$ \\
\hline Componente 2 & $\begin{array}{c}0.5943 \\
(0.48,0.88)\end{array}$ & $98.350 \%$ & $\begin{array}{c}3.3919 \\
(2.96,4.09)\end{array}$ & $86.630 \%$ & $\begin{array}{c}3.0930 \\
(2.51,4.44)\end{array}$ & $86.365 \%$ \\
\hline Componente 3 & $\begin{array}{c}0.0496 \\
(0.04,0.08)\end{array}$ & $100.000 \%$ & $\begin{array}{c}0.9828 \\
(0.86,1.19)\end{array}$ & $96.460 \%$ & $\begin{array}{c}0.5418 \\
(0.44,0.78)\end{array}$ & $91.785 \%$ \\
\hline Componente 4 & & & $\begin{array}{c}0.2282 \\
(0.20,0.28)\end{array}$ & $98.740 \%$ & $\begin{array}{c}0.3882 \\
(0.32,0.56)\end{array}$ & $95.668 \%$ \\
\hline Componente 5 & & & $\begin{array}{c}0.0951 \\
(0.08,0.11)\end{array}$ & $99.690 \%$ & $\begin{array}{c}0.2394 \\
(0.19,0.34)\end{array}$ & $98.062 \%$ \\
\hline Componente 6 & & & $\begin{array}{c}0.0182 \\
(0.01,0.02)\end{array}$ & $99.870 \%$ & $\begin{array}{c}0.1217 \\
(0.09,0.17)\end{array}$ & $99.280 \%$ \\
\hline Componente 7 & & & $\begin{array}{c}0.0083 \\
(0.007,0.010)\end{array}$ & $99.960 \%$ & $\begin{array}{c}0.0587 \\
(0.05,0.08)\end{array}$ & $99.866 \%$ \\
\hline Componente 8 & & & $\begin{array}{c}0.0026 \\
(0.002,0.003)\end{array}$ & $99.980 \%$ & $\begin{array}{c}0.0103 \\
(0.008,0.015)\end{array}$ & $99.969 \%$ \\
\hline Componente 9 & & & $\begin{array}{c}0.0013 \\
(0.001,0.002)\end{array}$ & $99.990 \%$ & $\begin{array}{c}0.0029 \\
(0.0024,0.0042)\end{array}$ & $99.999 \%$ \\
\hline Componente 10 & & & 0.0004 & $100.000 \%$ & $\begin{array}{c}0.0001 \\
(0.00008,0.00014)\end{array}$ & $100.000 \%$ \\
\hline
\end{tabular}

Fuente: elaboración propia con datos de Banxico. 
El primer componente principal presenta coeficientes positivos para los tres tramos del periodo 1978-2017, que sugiere la interpretación del primer componente principal como el "nivel" de la curva de rendimiento, ya que se puede visualizar como un "promedio ponderado", siendo que para 1978-1988 la tasa más relevante es CETES a 91 días. En tanto que para 19892017, las tasas más importantes son CETES a 364 días y Bonos a 3, 5 y 7 años.

El segundo componente principal cuenta con coeficientes positivos y negativos, lo que indica que las tasas de interés influyen de forma diferenciada a la pendiente de la curva de rendimiento. Durante el tramo 1989-2008 las tasas de interés CETES a 28, 91, 182 y 364 días influyen positivamente, en tanto que las tasas de los bonos a 3, 5, 7, 10, 20 y 30 años contribuyen negativamente. En contraste, en los años recientes 2009-2016 el comportamiento es diferente, pues son las tasas de los bonos a 3, 5, 7, 10, 20 y 30 años quienes contribuyen positivamente a la pendiente de la curva de rendimiento.

Empleando las variables estimadas, se realiza la prueba de causalidad en el sentido de Granger entre la tasa de crecimiento económico, la tasa de inflación, la brecha del PIB y los componentes principales de cada subperiodo. El Tabla 4 presenta con "palomita" el caso donde se rechazó la hipótesis nula y que aporta evidencia de causalidad entre las variables. El conjunto de todos los estadísticos de prueba y valores p se presentan en los anexos.

Con base en la tabla 4 se tiene evidencia para afirmar que el primer (C1) y segundo componente (C2) causan a la brecha del producto, a la tasa de inflación y a la tasa de crecimiento económico, aunque en menor medida, pues sólo se aprecia el resultado para 1989 en adelante. Este último hallazgo es similar a las estimaciones de Cerecero, Salazar y Salgado (2008) y Castellanos y Camero (2003), quienes realizaron ejercicios similares con los diferenciales de tasas de interés.

Tabla 4

Causalidad de Granger.

\begin{tabular}{|c|c|c|c|c|c|c|c|c|c|c|c|c|}
\hline \multicolumn{13}{|c|}{ Causalidad de Granger } \\
\hline & \multicolumn{4}{|c|}{$1978-1988$} & \multicolumn{4}{|c|}{ 1989-2008 } & \multicolumn{4}{|c|}{ 2009-2017 } \\
\hline Hipótesis nula & $\begin{array}{c}1 \\
\text { rezago }\end{array}$ & $\begin{array}{c}2 \\
\text { rezago }\end{array}$ & $\begin{array}{c}3 \\
\text { rezago }\end{array}$ & $\begin{array}{c}4 \\
\text { rezago }\end{array}$ & $\begin{array}{c}1 \\
\text { rezago }\end{array}$ & $\begin{array}{c}2 \\
\text { rezago }\end{array}$ & $\begin{array}{c}3 \\
\text { rezago }\end{array}$ & $\begin{array}{c}4 \\
\text { rezago }\end{array}$ & $\begin{array}{c}1 \\
\text { rezago }\end{array}$ & $\begin{array}{c}2 \\
\text { rezago }\end{array}$ & $\begin{array}{c}3 \\
\text { rezago }\end{array}$ & $\begin{array}{c}4 \\
\text { rezago }\end{array}$ \\
\hline BRECHA-C1 & $x$ & $x$ & $x$ & $x$ & $x$ & $x$ & $x$ & $x$ & $\checkmark$ & $x$ & $x$ & $\mathbf{x}$ \\
\hline C1-BRECHA & $\checkmark$ & $x$ & - & s & - & - & 。 & - & $x$ & 。 & 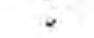 & - \\
\hline BRECHA-C2 & $x$ & $x$ & $x$ & 6 & $x$ & $x$ & $x$ & $x$ & $x$ & $x$ & $x$ & $x$ \\
\hline C2-BRECHA & $x$ & $x$ & $x$ & $x$ & . & 。 & . & • & $x$ & $x$ & $x$ & $x$ \\
\hline INFLACION-C1 & $x$ & $x$ & $x$ & $x$ & $x$ & $x$ & $x$ & $x$ & $x$ & $x$ & $x$ & $x$ \\
\hline C1-INFLACION & $x$ & $\checkmark$ & $x$ & $x$ & 。 & $\checkmark$ & $x$ & $\checkmark$ & $x$ & . & $\checkmark$ & $\checkmark$ \\
\hline INFLACION-C2 & $x$ & $x$ & 。 & 2 & $x$ & $x$ & $x$ & $\checkmark$ & $x$ & $x$ & $x$ & $x$ \\
\hline C2-INFLACION & , & 。 & 。 & 。 & $x$ & $x$ & $x$ & $x$ & $x$ & $x$ & $x$ & $x$ \\
\hline PIB-C1 & $x$ & $x$ & $x$ & $x$ & $x$ & $x$ & $x$ & $x$ & t & $x$ & $x$ & $x$ \\
\hline C1-PIB & $x$ & $x$ & $x$ & $x$ & $x$ & $\checkmark$ & 8 & $\checkmark$ & $x$ & $x$ & , & 2 \\
\hline PIB-C2 & $x$ & $x$ & $x$ & 2 & $x$ & $x$ & $x$ & $x$ & , & $x$ & $x$ & $x$ \\
\hline C2-PIB & $x$ & $x$ & $x$ & $x$ & $\therefore$ & 。 & 。 & $\checkmark$ & $x$ & $x$ & $x$ & $x$ \\
\hline
\end{tabular}

Fuente: elaboración propia con datos de Banxico. 
En la figura 4 (ver anexos) se presentan los diagramas de dispersión entre las variables BRECHA, INFLACION y PIB contra los componentes C1 y C2 para cada subperiodo, según la evidencia encontrada para la causalidad de Granger.

Los gráficos de la figura 4 intentan mostrar aquellos patrones que resultan visualmente directos, pues el argumento sólido se sitúa mediante las pruebas de causalidad de Granger y el vector autorregresivo, donde se considera la endogeneidad y temporalidad de las variables involucradas.

En la figura 4a (periodo 1978-1988) se puede observar que cuando C1 es negativo, BRECHA se incrementa y cuando $\mathrm{C} 1$ es positivo, BRECHA disminuye. Mayores niveles de la variable C1 tienden a cerrar la brecha del PIB. En cambio, cuando C1 se incrementa, INFLACION tiende a subir. La variable PIB tiende a bajar cuando C2 se incrementa. Dada la interpretación usual de C1 (Martínez y Núñez, 2012), los gráficos sugieren que el nivel de la curva de rendimiento afecta positivamente a la tasa de inflación. Mientras que el componente $\mathrm{C} 2$, asociado a la pendiente de la curva de rendimiento, afecta negativamente a la variable PIB.

En la figura 4b (periodo 1989-2008) la variable BRECHA se relaciona de manera inversa con $\mathrm{C} 1$, mientras que $\mathrm{C} 1$ afecta de forma directa y positiva a INFLACION. El componente C2 también afecta positivamente a la tasa de inflación y a la tasa de crecimiento económico.

En la figura 4.c del anexo se puede observar que el segundo componente tiene una relación positiva con la tasa de crecimiento económico. Esto señala que mientras mayor sea la tasa de vencimiento en el largo plazo, en relación a las tasas de corto plazo, existe un efecto positivo. En el caso de BRECHA, se aprecia que conforme aumenta $\mathrm{C} 1$ se cierra la brecha de producto y la tasa de crecimiento del PIB disminuye. Sin embargo, se mantiene una relación positiva con la variable INFLACION.

La Tabla 5 presenta los coeficientes estimados de los componentes principales dentro de un modelo VAR que relaciona endógenamente a las variables macroeconómicas con $\mathrm{C} 1$ y C2, ver anexo. El número de rezagos óptimo, según los criterios de información de Akaike y Schwarz, se reportan en los anexos y son iguales a cuatro trimestres.

Los coeficientes estimados (tabla 5) representan, en cada caso, el impacto que la variación del componente principal ejerce sobre la tasa de crecimiento económico, la brecha de PIB y la tasa de inflación. Se puede apreciar que el componente principal que se encuentra asociado al nivel de la curva de rendimiento $(\mathrm{C} 1)$ presenta un impacto negativo significativo sobre PIB, lo que intuitivamente señala que si el nivel de las tasas de interés sube, existe un efecto inverso sobre la tasa de crecimiento económico. En contraste, un cambio de una unidad en el componente principal asociado a la pendiente de la curva de rendimiento $(\mathrm{C} 2)$, ejerce un efecto positivo, lo que indica que a mayor diferencial entre las tasas de interés de corto y largo plazo, existe un efecto positivo sobre la tasa de crecimiento económico. Este último resultado es similar a lo señalado por Cerecero, Salazar y Salgado (2008) y Castellanos y Camero (2003).

En relación al impacto sobre la variable BRECHA se tiene que existe evidencia de causalidad de Granger en los tres subperiodos de crecimiento estacionario, aunque sobresale el periodo de 1989-2008, donde ambos componentes son relevantes a lo largo de cuatro trimestres de rezago, ver tabla 4. Si se consideran los coeficientes del modelo VAR, se tienen coeficientes positivos y negativos. Dado que la variable BRECHA se define como el cambio porcentual entre el PIB potencial y el PIB real del periodo, entonces los componentes C1 y C2 cierran la brecha del producto, ver tabla 5. 
En el caso de la variable INFLACION, se aprecia también signos positivos y negativos de los coeficientes de los componentes $\mathrm{C}$ y $\mathrm{C} 2$, ver tabla 5 . No obstante, el efecto más grande es de carácter positivo, así que los componentes C1 y C2 contribuyen hacia arriba a la tasa de inflación. Asimismo, si se observa la tabla 4 se tiene evidencia de causalidad en el sentido de Granger, particularmente en el subperiodo 1978-1988.

En general, para las tres variables BRECHA, INFLACION y PIB se ha encontrado evidencia de causalidad en el sentido de Granger debido a $\mathrm{C} 1$ y $\mathrm{C} 2$, además bajo el modelo VAR existe evidencia de impactos significativos de los componentes principales, que están asociados al nivel y pendiente de la curva de rendimiento.

Tabla 5

Coeficientes del modelo VAR en 1978-2017.

\begin{tabular}{|c|c|c|c|c|c|}
\hline & PIB & INFLACION & BRECHA & $\mathrm{C} 1$ & $\mathrm{C} 2$ \\
\hline $\mathrm{C} 1(-1)$ & $\begin{array}{c}-0.0107 \\
(0.0049) \\
{[-2.1944]}\end{array}$ & $\begin{array}{c}0.0194 \\
(0.007) \\
{[2.7871]}\end{array}$ & $\begin{array}{c}-0.0118 \\
(0.0049) \\
{[-2.4218]}\end{array}$ & $\begin{array}{c}1.2224 \\
(0.1041) \\
{[11.7417]}\end{array}$ & $\begin{array}{c}-0.0226 \\
(0.0489) \\
{[-0.4624]}\end{array}$ \\
\hline $\mathrm{C} 1(-2)$ & $\begin{array}{c}0.0118 \\
(0.0069) \\
{[1.708]}\end{array}$ & $\begin{array}{c}-0.0180 \\
(0.0099) \\
{[-1.8204]}\end{array}$ & $\begin{array}{c}0.0141 \\
(0.0069) \\
{[2.0339]}\end{array}$ & $\begin{array}{c}-0.6999 \\
(0.1481) \\
{[-4.7272]}\end{array}$ & $\begin{array}{c}0.1269 \\
(0.0695) \\
{[1.8252]}\end{array}$ \\
\hline $\mathrm{C} 1(-3)$ & $\begin{array}{c}-0.0093 \\
(0.007) \\
{[-1.3399]}\end{array}$ & $\begin{array}{c}0.0255 \\
(0.01) \\
{[2.5639]}\end{array}$ & $\begin{array}{c}-0.0101 \\
(0.007) \\
{[-1.4536]}\end{array}$ & $\begin{array}{c}0.6083 \\
(0.1491) \\
{[4.0805]}\end{array}$ & $\begin{array}{c}0.0148 \\
(0.07) \\
{[0.212]}\end{array}$ \\
\hline $\mathrm{C} 1(-4)$ & $\begin{array}{c}0.0033 \\
(0.0048) \\
{[0.6996]}\end{array}$ & $\begin{array}{c}-0.0147 \\
(0.0068) \\
{[-2.155]}\end{array}$ & $\begin{array}{c}0.0038 \\
(0.0048) \\
{[0.7994]}\end{array}$ & $\begin{array}{c}-0.2090 \\
(0.1019) \\
{[-2.0517]}\end{array}$ & $\begin{array}{c}-0.0624 \\
(0.0478) \\
{[-1.305]}\end{array}$ \\
\hline $\mathrm{C} 2(-1)$ & $\begin{array}{c}0.0015 \\
(0.0086) \\
{[0.1739]}\end{array}$ & $\begin{array}{c}0.0908 \\
(0.0123) \\
{[7.4146]}\end{array}$ & $\begin{array}{c}0.0006 \\
(0.0086) \\
{[0.0749]}\end{array}$ & $\begin{array}{c}1.8711 \\
(0.1834) \\
{[10.2037]}\end{array}$ & $\begin{array}{c}0.2372 \\
(0.0861) \\
{[2.7556]}\end{array}$ \\
\hline $\mathrm{C} 2(-2)$ & $\begin{array}{c}0.0269 \\
(0.0115) \\
{[2.3341]}\end{array}$ & $\begin{array}{c}-0.0230 \\
(0.0165) \\
{[-1.3959]}\end{array}$ & $\begin{array}{c}0.0294 \\
(0.0115) \\
{[2.5513]}\end{array}$ & $\begin{array}{c}-1.1463 \\
(0.2471) \\
{[-4.6384]}\end{array}$ & $\begin{array}{c}0.1713 \\
(0.116) \\
{[1.4766]}\end{array}$ \\
\hline $\mathrm{C} 2(-3)$ & $\begin{array}{c}-0.0022 \\
(0.0122) \\
{[-0.1823]}\end{array}$ & $\begin{array}{c}0.0468 \\
(0.0175) \\
{[2.6801]}\end{array}$ & $\begin{array}{c}-0.0034 \\
(0.0122) \\
{[-0.2819]}\end{array}$ & $\begin{array}{c}0.6100 \\
(0.2613) \\
{[2.3347]}\end{array}$ & $\begin{array}{c}-0.2102 \\
(0.1227) \\
{[-1.7136]}\end{array}$ \\
\hline $\mathrm{C} 2(-4)$ & $\begin{array}{c}-0.0175 \\
(0.0117) \\
{[-1.49]}\end{array}$ & $\begin{array}{l}-0.0367 \\
(0.0168) \\
{[-2.185]}\end{array}$ & $\begin{array}{c}-0.0112 \\
(0.0117) \\
{[-0.951]}\end{array}$ & $\begin{array}{c}-1.0496 \\
(0.2514) \\
{[-4.1759]}\end{array}$ & $\begin{array}{c}-0.3132 \\
(0.118) \\
{[-2.6543]}\end{array}$ \\
\hline
\end{tabular}

Fuente: elaboración propia con datos de Banxico.

***: nivel de significancia al $10 \%$

***: nivel de significancia al $5 \%$

***: nivel de significancia al $1 \%$ 
Ahora bien, en la tabla 6 se presenta la descomposición de varianza del modelo VAR, donde se puede apreciar que los componentes $\mathrm{C} 1$ y C2 contribuyen a la variación del PIB, BRECHA e INFLACION en al menos 2\%, dados los trimestres 1, 4, 8 y 12 de la estimación. En los anexos se muestran el conjunto total de estimaciones y se observa el mismo comportamiento para el resto de los trimestres del periodo 1978-2017.

\section{Tabla 6}

Descomposición de varianza.

\begin{tabular}{|c|c|c|c|c|c|c|}
\hline \multicolumn{7}{|c|}{ Descomposición de la varianza: PIB } \\
\hline Periodo & Error estándar & PIB & INFLACION & BRECHA & $\mathrm{C} 1$ & $\mathrm{C} 2$ \\
\hline 1 & 0.0154 & 100 & 0.0000 & 0.0000 & 0.0000 & 0.0000 \\
\hline 4 & 0.0171 & 89.9307 & 4.2836 & 2.7928 & 2.9155 & 0.0774 \\
\hline 8 & 0.0203 & 85.9748 & 4.3048 & 2.8316 & 3.5114 & 3.3773 \\
\hline 12 & 0.0219 & 85.5181 & 4.3998 & 2.7579 & 3.4910 & 3.8332 \\
\hline \multicolumn{7}{|c|}{ Descomposición de la varianza: INFLACION } \\
\hline Periodo & Error estándar & PIB & INFLACION & BRECHA & $\mathrm{C} 1$ & $\mathrm{C} 2$ \\
\hline 1 & 0.0154 & 100 & 0.0000 & 0.0000 & 0.0000 & 0.0000 \\
\hline 4 & 0.0171 & 89.9307 & 4.2836 & 2.7928 & 2.9155 & 0.0774 \\
\hline 8 & 0.0203 & 85.9748 & 4.3048 & 2.8316 & 3.5114 & 3.3773 \\
\hline 12 & 0.0219 & 85.5181 & 4.3998 & 2.7579 & 3.4910 & 3.8332 \\
\hline \multicolumn{7}{|c|}{ Descomposición de la varianza: BRECHA } \\
\hline Periodo & Error estándar & PIB & INFLACION & BRECHA & $\mathrm{C} 1$ & $\mathrm{C} 2$ \\
\hline 1 & 0.0154 & 100 & 0.0000 & 0.0000 & 0.0000 & 0.0000 \\
\hline 4 & 0.0171 & 89.9307 & 4.2836 & 2.7928 & 2.9155 & 0.0774 \\
\hline 8 & 0.0203 & 85.9748 & 4.3048 & 2.8316 & 3.5114 & 3.3773 \\
\hline 12 & 0.0219 & 85.5181 & 4.3998 & 2.7579 & 3.4910 & 3.8332 \\
\hline
\end{tabular}

Fuente: elaboración propia con datos de Banxico.

En términos gráficos, la figura 2 muestra las funciones impulso-respuesta para las variables macroeconómicas y los componentes $\mathrm{C} 1$ y C2. Se aprecia que para el caso de la variable BRECHA, la respuesta es menor a cero y los componentes contribuyen a cerrar la brecha del producto.

Cuando se observan las gráficas para la variable INFLACION, se tiene un efecto positivo por parte de $\mathrm{C} 1$, que contribuye a elevar la tasa de inflación a lo largo de los 12 trimestres señalados. En tanto que C2 eleva la tasa de inflación en el corto plazo, pero influye negativamente hacia el largo plazo. Es decir, el componente $\mathrm{C} 1$, asociado a la pendiente de la curva de rendimiento, contribuye a subir la tasa de inflación en el corto plazo y a bajarla hacia el largo plazo.

Finalmente, el efecto del componente $\mathrm{C} 1$ sobre la tasa de crecimiento económico es oscilatorio, pues la respuesta de PIB es subir y bajar alrededor del punto inicial, aunque sesgado hacia abajo, es decir, un incremento en $\mathrm{C} 1$ tiende a disminuir la variable PIB. En tanto que el componente C2 eleva ligeramente a la variable PIB, después se aprecia una caída y en el largo plazo un efecto positivo. 

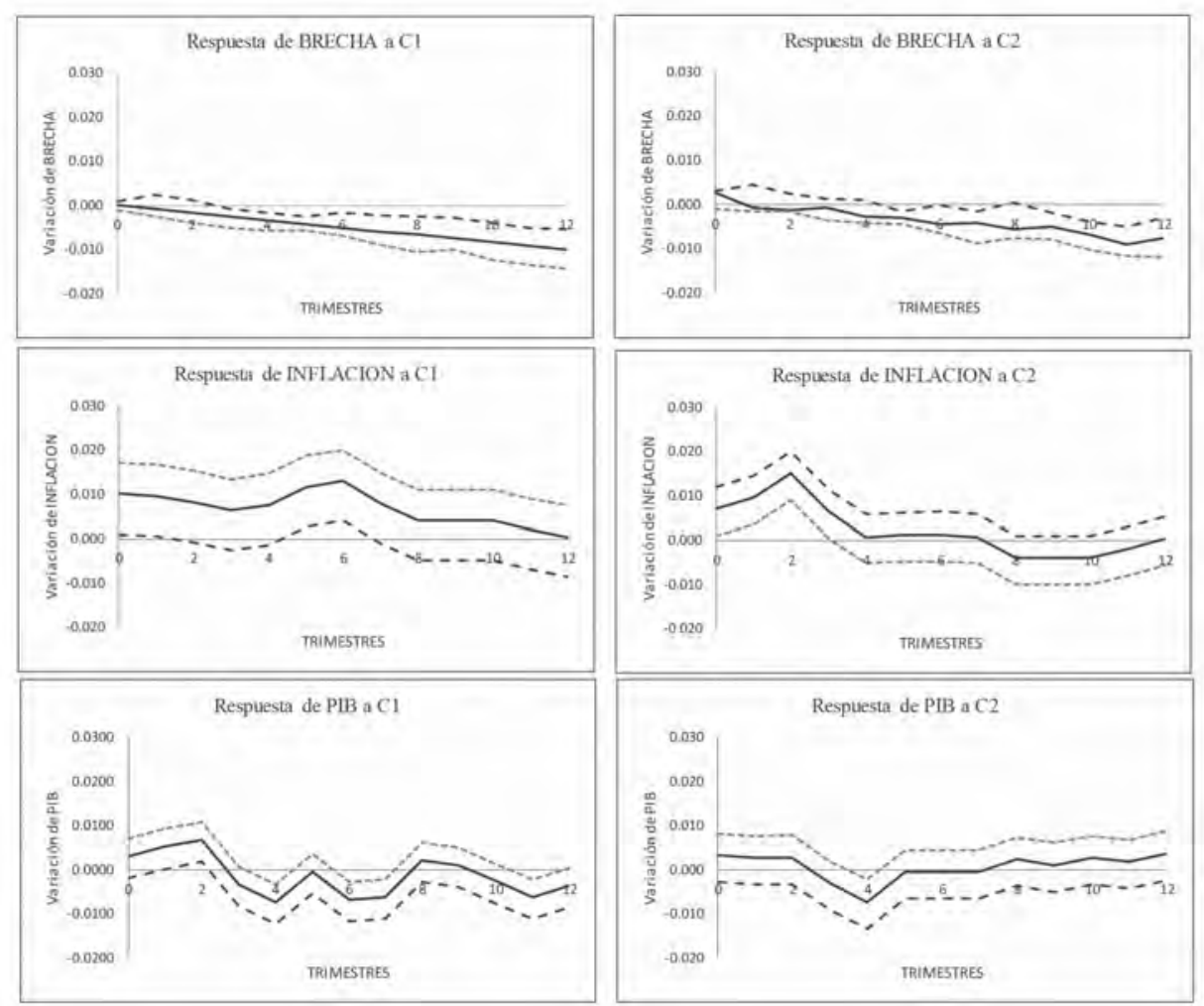

Figura 2 Funciones impulso-respuesta.

Fuente: elaboración propia con datos de Banxico.

\section{Conclusiones}

En este trabajo se encuentra evidencia para afirmar que la dinámica de la curva de rendimiento se puede estudiar mediante dos variables (componentes principales) para cada uno de los tres subperiodos 1978-1988, 1989-2008 y 2009-2017. Los factores encontrados concuerdan con los citados en la literatura y que usualmente se asocian con el nivel y la pendiente de la curva de rendimiento, como se tiene en Martínez y Núñez (2012), Cortés, Ramos y Torres (2009) y Jiménez (2002).

En el subperiodo 1978-1988 sólo el primer componente principal es relevante y explica $78.54 \%$ de la variación de las tasas de interés, todos los coeficientes son mayores a cero. En este caso, ACP sólo encuentra como relevante el nivel de la curva de rendimiento, según el criterio de simulación paralela, ver figura 3 en el anexo.

En los años 1989-2008 y 2009-2016 resultan significativos los dos primeros componentes principales, reúnen $86.63 \%$ y $86.37 \%$ de la variación de las tasas de interés, respectivamente. En estos periodos, tanto el nivel como la pendiente de la curva de rendimiento son relevantes, siendo que las tasas de los bonos a 3, 5, 7, 10, 20 y 30 años contribuyen positivamente a la pendiente de la curva en 2009-2017, en contraste al subperiodo 1989-2008.

En cada subperiodo se encontró evidencia de causalidad de Granger de los componentes C1 y C2 sobre las variables tasa de crecimiento económico, tasa de inflación y brecha de 
producto. Estos resultados se ratifican en el modelo VAR estimado, donde se aprecian efectos diferenciados de los componentes $\mathrm{C} 1$ y $\mathrm{C} 2$. Mediante las funciones impulso-respuesta se encuentra que los componentes $\mathrm{C} 1$ y $\mathrm{C} 2$ contribuyen a cerrar la brecha del producto. En tanto que para el caso de la variable INFLACION, la variable $\mathrm{C} 1$ contribuye positivamente mientras que C2 eleva la tasa de inflación en el corto plazo, pero influye negativamente hacia el largo plazo. Ahora bien, cuando nos fijamos en la tasa de crecimiento económico, el efecto del componente $\mathrm{C} 1$ es oscilatorio, aunque sesgado hacia abajo, es decir, un incremento en $\mathrm{C} 1$ tiende a disminuir la variable PIB, pero al mismo tiempo el componente C2 eleva la variable PIB en un horizonte de largo plazo.

Este último resultado concuerda con el efecto positivo que tiene un cambio en la pendiente de la curva de rendimiento sobre la actividad económica, como se señala en Cerecero, Salazar y Salgado (2008) y Castellanos y Camero (2003).

Finalmente, se puede señalar que los efectos de $\mathrm{C} 1$ y $\mathrm{C} 2$ reflejan un conjunto de factores latentes, cuyo movimiento es predictor de la actividad económica. En este sentido, el ejercicio presentado en este documento intenta construir un puente entre el sector real de la economía y los movimientos de las variables financieras.

\section{Referencias}

Ang, A. y Piazzesi, M. (2003). A No-Arbitrage Vector Autoregression of Term Structure Dynamics with macroeconomics and Latent variables. Journal of Monetary Economics, 50, 745-787. https://doi.org/10.1016/s03043932(03)00032-1

Barber, J. y Cooper, M. (1996). Immunization Using Principal Component Analysis. Journal of Portfolio Management, 23 (1), 99-105. https://doi.org/10.3905/jpm.1996.409574

Bühler, A. y Zimmermann (1996). A statistical analysis of the term structure of interest rates in Switzerland and Germany, Journal of Fixed Income, 6(3), 55-67. https://doi.org/10.3905/jifi.1996.408182

Castellanos, S.G. y Camero, E. (2003). La estructura temporal de las tasas de interés en México: ¿puede ésta predecir la actividad económica futura? Revista de Análisis Económico, 18 (2), 37-52.

Disponible en: http://repositorio.uahurtado.cl/handle/11242/1790 Consultado: 13/05/2016

Cerecero, M., Salazar, C. D. y Salgado, B. H. (2008). La curva de rendimiento y su relación con la actividad económica: una aplicación para México. Working paper, Banco de México.

Disponible en: https://www.econstor.eu/handle/10419/83775 Consultado: 15/04/2016

Cortés, J., Ramos M. y Torres A. (2009). An Empirical Analysis of the Mexican Term Structure of Interest Rate. Economics Bulletin, 29 (3), 2310-2323.

Disponible en: https://core.ac.uk/download/pdf/6442917.pdf Consultado: 27/03/2016

Cuadras, C. (2014). Nuevos métodos de análisis multivariante, Manacor, Barcelona, España. Disponible en: http://www.ub.edu/stat/personal/cuadras/metodos.pdf Consultado: 12/05/2016

D’Ecclesia, R.L. and Zenios, S. A. (1994). Risk Factor Analysis and Portfolio Immunization in the Italian Bond Market. Journal of Fixed Income 4(29), 51-58. https://doi.org/10.3905/jfi.1994.408113

Dinno, A. (2009). Implementing Horn's parallel analysis for principal component analysis and factor analysis. Stata Journal, 9 (2), 291-302.

Disponible en: https://www.stata-journal.com/article.html?article=st0166 Consultado: 11/06/2016

Enders, W. (2004). Applied Econometric Time Series, 2a. ed., John Wiley \& Sons, Inc.

García, V. S. (2011). Algunas consideraciones sobre la estructura temporal de tasas de interés del gobierno en México (No. 2011-18). Working Papers, Banco de México. Disponible en: https://www.econstor.eu/handle/10419/83698 Consultado: 10/03/2016 
Greene, W.H. (2010). Econometric Analysis, 5a. ed., Prentice Hall, New Jersey.

Jiménez, V. (2002) Testing the stability of the Components Explaining Changes of the Yield Curve in Mexico. A principal Component Analysis Approach. Trans 27th ICA.

Disponible en: https://pdfs.semanticscholar.org/7760/5bb62c251124c19968e2f883d12d5816c850.pdf

Consultado: 17/02/2016

Johnson, R. y Wichern, D. (2000). Applied multivariate statistical analysis. Vol. 5. Englewood Cliffs, NJ: Prentice Hall.

Knez, J., Litterman R. y Scheinkman, J. (1996). Exploration into Factors Explaining Money Market Returns. Journal of Finance, 49(5), 1861-1881. https://doi.org/10.2307/2329274

Lardic, S., Priaulet, P. y Priaulet, S. (2003). PCA of the Yield Curve Dynamics: Questions of Methodologies, Journal of Bond Trading and Management, 1(4), 327-349.

Litterman, R. y Scheinkman, J. (1991). Common Factors Affecting Bond Returns. Journal of Fixed Income, 1, 54-61. https://doi.org/10.3905/jfi.1991.692347

Martínez, C. y Núñez, J.A. (2012). Análisis de componentes principales de la estructura a plazos de las tasas de interés en México. eseconomia, VII (33), 3-23. Disponible en: http://yuss.me/revistas/ese/ese2012v07n33a01p003_023. pdf. Consultado: 14/04/2016

Mardia, K. V., Kent, J. T. y Bibby, J. M. (1979). Multivariate analysis. 1a. ed., New York, Academy Press.

Noriega, A. y Rodríguez-Peréz, C.A. (2011). Estacionariedad, Cambios Estructurales y Crecimiento Económico en México: 1895-2008. Documento de Trabajo, Banco de México.

Redington, F.M. (1952). Review of the Principle of Life Office Valuations. Journal of the Institute of Actuaries, 78 (1), 286-340.

\section{Anexos}

\section{A. Componentes principales y simulación paralela, 1978-2017}

\begin{tabular}{|c|c|c|c|c|c|c|}
\hline \multirow[b]{3}{*}{ DCETES28 } & \multicolumn{2}{|c|}{ 1978-1988 } & \multicolumn{2}{|c|}{$1989-2008$} & \multicolumn{2}{|c|}{$2009-2017$} \\
\hline & Componente 1 & Componente 2 & Componente 1 & Componente 2 & Componente 1 & Componente 2 \\
\hline & $\begin{array}{c}0.596 \\
(0.573,0.619)\end{array}$ & $\begin{array}{c}-0.559 \\
(-0.629,-0.587)\end{array}$ & $\begin{array}{c}0.134 \\
(0.132,0.135)\end{array}$ & $\begin{array}{c}0.502 \\
(0.485,0.518)\end{array}$ & $\begin{array}{c}0.282 \\
(0.277,0.288)\end{array}$ & $\begin{array}{c}-0.386 \\
(-0.396,-0.377)\end{array}$ \\
\hline DCETES91 & $\begin{array}{c}0.626 \\
(0.601,0.651)\end{array}$ & $\begin{array}{c}-0,233 \\
(-0,301,-0.217)\end{array}$ & $\begin{array}{c}0.171 \\
(0.169,0.173)\end{array}$ & $\begin{array}{c}0.485 \\
(0.470,0.501)\end{array}$ & $\begin{array}{c}0.306 \\
(0.301,0.313)\end{array}$ & $\begin{array}{c}-0.380 \\
(-0,388,-0,370)\end{array}$ \\
\hline DCETES182 & $\begin{array}{c}0.504 \\
(0.487,0.52)\end{array}$ & $\begin{array}{c}0.796 \\
(0.564,0.897)\end{array}$ & $\begin{array}{c}0.268 \\
(0.264,0.273)\end{array}$ & $\begin{array}{c}0.419 \\
(0.408,0.431)\end{array}$ & $\begin{array}{c}0,334 \\
(0.327,0.341)\end{array}$ & $\begin{array}{c}-0.345 \\
(-0.353,-0.338)\end{array}$ \\
\hline DCETES364 & & & $\begin{array}{c}0.334 \\
(0.327,0.341)\end{array}$ & $\begin{array}{c}0.322 \\
(0.315,0.329)\end{array}$ & $\begin{array}{c}0.353 \\
(0.346,0.362)\end{array}$ & $\begin{array}{c}-0.295 \\
(-0.298,-0.287)\end{array}$ \\
\hline DBONOS3 & & & $\begin{array}{c}0,381 \\
(0,371,0.390)\end{array}$ & $\begin{array}{c}0.098 \\
(-0.099,-0.097)\end{array}$ & $\begin{array}{c}0.365 \\
(0.356,0.373)\end{array}$ & $\begin{array}{c}0.049 \\
(0,049,0.049)\end{array}$ \\
\hline DBONOSS & & & $\begin{array}{c}0.393 \\
(0.383,0.403)\end{array}$ & $\begin{array}{c}-0.137 \\
(-0.139,-0.136)\end{array}$ & $\begin{array}{c}0.349 \\
(0.342,0.358)\end{array}$ & $\begin{array}{c}0.150 \\
(0.148,0.151)\end{array}$ \\
\hline DBONOS7 & & & $\begin{array}{c}0.401 \\
(0.391,0.412)\end{array}$ & $\begin{array}{c}-0.144 \\
(-0.146,-0.143)\end{array}$ & $\begin{array}{c}0.361 \\
(0.352,0.369)\end{array}$ & $\begin{array}{c}0.274 \\
(0.270,0.279)\end{array}$ \\
\hline DBONOS10 & & & $\begin{array}{c}0.393 \\
(0.383,0.403)\end{array}$ & $\begin{array}{c}-0.166 \\
(-0.168,-0.165)\end{array}$ & $\begin{array}{c}0.276 \\
(0.269,0.279)\end{array}$ & $\begin{array}{c}0.323 \\
(0.317,0.330)\end{array}$ \\
\hline DBONOS20 & & & $\begin{array}{c}0.283 \\
(0.277,0.288)\end{array}$ & $\begin{array}{c}-0.274 \\
(-0.279,-0.269)\end{array}$ & $\begin{array}{c}0.300 \\
(0.294,0.305)\end{array}$ & $\begin{array}{c}0.342 \\
(0.336,0.351)\end{array}$ \\
\hline DBONOS30 & & & $\begin{array}{c}0.273 \\
(0.268,0.278)\end{array}$ & $\begin{array}{c}0.285 \\
(-0.29,-0.279)\end{array}$ & $\begin{array}{c}0.198 \\
(0.195,0.200)\end{array}$ & $\begin{array}{c}0.423 \\
(0.412,0.435)\end{array}$ \\
\hline
\end{tabular}

Fuente: elaboración propia con datos de Banxico. 

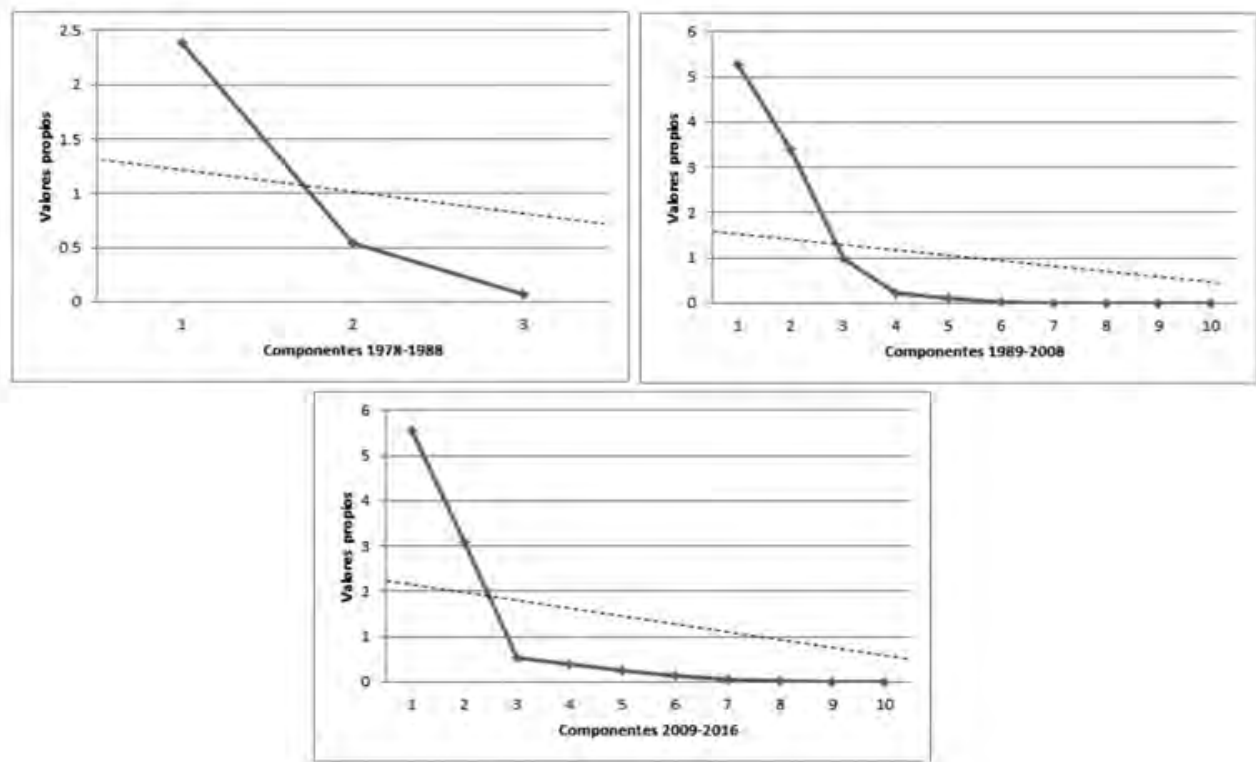

Figura 3

Simulación paralela 1978-2017.

Fuente: elaboración propia con datos de Banxico.

\section{B. Pruebas de causalidad de Granger.}

\begin{tabular}{|c|c|c|c|c|c|c|c|c|}
\hline \multicolumn{9}{|c|}{ Causalidad de Granger: 1 rezago } \\
\hline \multicolumn{3}{|c|}{ 1978-1988 } & \multicolumn{3}{|c|}{$1989-2008$} & \multicolumn{3}{|c|}{$2009-2017$} \\
\hline Hipótesis nula & Estadistico F & alorp & Hipótesis nula & Estadistico & alor $p$ & Hipótesis nula & istadistico & alor $p$ \\
\hline BRECHA-C1 & 0.9722 & 0.3301 & BRECHA-C1 & 0.0622 & 0.8037 & BRECHA-CI & 15.3815 & 0.0005 \\
\hline C1-BRECHA & 35.9317 & 0.0000 & C1-BRECHA & 8.8610 & 0,0039 & C1-BRECHA & 2.5148 & 0.1229 \\
\hline BRECHA-C2 & 0.7895 & 0.3796 & BRECHA-C2 & 2.1099 & 0.1504 & BRECHA-C2 & 0.8677 & 0.3588 \\
\hline C2-BRECHA & 0.0658 & 0.7988 & C2-BRECHA & 8.2687 & 0.0052 & C2-BRECHA & 0.1059 & 0.7471 \\
\hline INFLACION-C1 & 0.4740 & 0,4951 & INFLACION-C1 & 1.1895 & 0.2788 & INFLACION-CI & 0.9919 & 0.3270 \\
\hline C1-INFLACION & 2.5267 & 0.1198 & C1-INFLACION & 6.1084 & 0.0157 & C1-INFLACION & 0.0002 & 0.9877 \\
\hline INFLACION-C2 & 1.1669 & 0.2865 & INFLACION-C2 & 0.3733 & 0.5430 & INFLACION-C2 & 0.0130 & 0.9099 \\
\hline C2-INFLACION & 55.5878 & 0.0000 & C2-INFLACION & 0.1106 & 0.7403 & C2-INFLACION & 0.0182 & 0.8937 \\
\hline PIB-C1 & 2.6708 & 0.1101 & PIB-C1 & 0.4088 & 0.5245 & PIB-C1 & 3.0280 & 0.0918 \\
\hline C1-PIB & 0.3328 & 0.5673 & C1.PIB & 0.0005 & 0.9433 & C1-PIB & 1.0125 & 0.3221 \\
\hline PIB-C2 & 0.0592 & 0.8091 & PIB-CZ & 0.0576 & 0.8110 & PIB-C2 & 5.5648 & 0.0248 \\
\hline C. 2 PIB & 0.0072 & 0.9330 & $\mathrm{CZ}_{2} \cdot \hat{\mathrm{P}} \mathrm{B}$ & 13.9274 & 0.0004 & CZ-P̂IB & 0.2791 & 0.6011 \\
\hline
\end{tabular}

Fuente: elaboración propia con datos de Banxico. 

http://dx.doi.org/10.22201/fca.24488410e.2018.1204

\begin{tabular}{|c|c|c|c|c|c|c|c|c|}
\hline \multicolumn{9}{|c|}{ Causalidad de Granger: 2 rezagos } \\
\hline \multicolumn{3}{|c|}{ 1978-1988 } & \multicolumn{3}{|c|}{$1989-2008$} & \multicolumn{3}{|c|}{$2009-2017$} \\
\hline Hipótesis nula & Estadistica F & alorp & Hipótesis nula & Estadistico & alor $p$ & Hipótesis nula & stadístico & torp \\
\hline BRECHA-CI & 0.8693 & 0.4276 & BRECHA-CI & 0.2228 & 0.8008 & BRECHA-C1 & 2,2590 & 0.1225 \\
\hline C1-BRECHA & 2.0276 & 0.1460 & C1-BRECHA. & 9.9806 & 0.0001 & C1-BRECHA & 3.1383 & 0.0584 \\
\hline BRECHA-C2 & 0.6781 & 0.5138 & BRECHA-C2 & 0.8879 & 0.4158 & BRECHA-C2 & 1.8234 & 0.1795 \\
\hline C2-BRECHA & 0.1816 & 0.8347 & C2-BRECHA & 9.3441 & 0.0002 & C2-BRECHA & 1.6720 & 0.2055 \\
\hline INFLACION-CI & 1.1194 & 0.3373 & INFLACION-CI & 0.9318 & 0.3984 & INFLACION-CI & 0.7465 & 0.4829 \\
\hline CI-INFLACION & 2.6453 & 0.0844 & CI-INFLACION & 4.4844 & 0.0145 & CI-INFLACION & 2.9405 & 0.0687 \\
\hline INFLACION-C2 & 1.0900 & 0.3468 & INFLACION-C2 & 2.0422 & 0.1369 & INFLACION-C2 & 1.5773 & 0.2237 \\
\hline CZ-INFLACION & 23.7165 & 0.0000 & C2-INFLACION & 0.3045 & 0.7384 & C2-INFLACION & 0.4619 & 0.6347 \\
\hline $\mathrm{PIB}-\mathrm{Cl}$ & 1.4995 & 0.2365 & PIB-CI & 0.3637 & 0,6963 & $\mathrm{PIB}-\mathrm{Cl}$ & 2.2338 & 0.1252 \\
\hline CL-PIB & 0.2953 & 0.7461 & C1-PIB & 9.7559 & 0.0002 & C1-PIB & 0.8714 & 0.4290 \\
\hline $\mathrm{PIB}-\mathrm{C} 2$ & 0.0860 & 0.9178 & PIB-C2 & 0.0934 & 0.9109 & $\mathrm{PIB}-\mathrm{C} 2$ & 2.2972 & 0.1185 \\
\hline C2-PIB & 0.0519 & 0.9495 & C2-PIB & 7.1049 & 0.0025 & CZ-PIB & 2.3835 & 0.1101 \\
\hline
\end{tabular}

Fuente: elaboración propia con datos de Banxico.

\begin{tabular}{|c|c|c|c|c|c|c|c|c|}
\hline \multicolumn{9}{|c|}{ Causalidad de Granger: 3 rezagos } \\
\hline \multicolumn{3}{|c|}{$1978-1988$} & \multicolumn{3}{|c|}{$1989-2008$} & \multicolumn{3}{|c|}{ 2009-2017 } \\
\hline Hipótesis nula & Estadístico $\mathrm{F}$ & Valor $p$ & Hipótesis nula & Estadístico & alor $\mathrm{p}$ & Hipótesis nula & Estadístico & alor $\mathrm{p}$ \\
\hline BRECHA-C1 & 0.6506 & 0.5881 & BRECHA-C1 & 0.1513 & 0.9286 & BRECHA-C1 & 1.4325 & 0.2551 \\
\hline C1-BRECHA & 2.3785 & 0.0869 & C1-BRECHA & 6.9721 & 0.0003 & C1-BRECHA & 2.6485 & 0.0691 \\
\hline BRECHA-C2 & 0.6560 & 0.5848 & BRECHA-C2 & 0.4032 & 0.7511 & BRECHA-C2 & 1.5012 & 0.2367 \\
\hline C2-BRECHA & 0.1329 & 0.9398 & C2-BRECHA & 5.9197 & 0.0011 & C2-BRECHA & 1.6723 & 0.1964 \\
\hline INFLACION-C1 & 0.6101 & 0.6131 & INFLACION-C1 & 0.3570 & 0.7842 & INFLACION-C1 & 1.1759 & 0.3373 \\
\hline C1-INFLACION & 1.4871 & 0.2355 & C1-INFLACION & 1.0179 & 0.3898 & C1-INFLACION & 6.2737 & 0.0023 \\
\hline INFLACION-C2 & 3.4626 & 0.0268 & INFLACION-C2 & 1.1257 & 0.3444 & INFLACION-C2 & 0.9877 & 0.4133 \\
\hline C2-INFLACION & 14.3284 & 0.0000 & C2-INFLACION & 0.2710 & 0.8461 & C2-INFLACION & 0.5136 & 0.6763 \\
\hline PIB-C1 & 0.8236 & 0.4900 & PIB-C1 & 0.4867 & 0.6926 & PIB-C1 & 1.7420 & 0.1821 \\
\hline C1-PIB & 0.2859 & 0.8352 & C1-PIB & 7.5425 & 0.0002 & C1-PIB & 2.3443 & 0.0953 \\
\hline PIB-C2 & 0.0986 & 0.9602 & PIB-C2 & 0.3753 & 0.7711 & PIB-C2 & 1.5029 & 0.2362 \\
\hline $\mathrm{C} 2-\mathrm{PIB}$ & 0.0464 & 0.9865 & C2-PIB & 5.6100 & 0.0016 & C2-PIB & 0.6663 & 0.5801 \\
\hline
\end{tabular}

Fuente: elaboración propia con datos de Banxico.

\begin{tabular}{|c|c|c|c|c|c|c|c|c|}
\hline \multicolumn{9}{|c|}{ Causalidad de Granger: 4 rezagos } \\
\hline \multicolumn{3}{|c|}{ 1978-1988 } & \multicolumn{3}{|c|}{$1989-2008$} & \multicolumn{3}{|c|}{ 2009-2017 } \\
\hline Hipótesis nula & Estadístico $\mathrm{F}$ & Valor $p$ & Hipótesis nula & Estadístico & alor $\mathrm{p}$ & Hipótesis nula & Estadístico & alor $\mathrm{p}$ \\
\hline BRECHA-C1 & 0.9894 & 0.4279 & BRECHA-C1 & 0.3322 & 0.8554 & BRECHA-C1 & 1.2188 & 0.3279 \\
\hline C1-BRECHA & 2.3707 & 0.0740 & C1-BRECHA & 4.9802 & 0.0014 & C1-BRECHA & 17.6800 & 0.0000 \\
\hline BRECHA-C2 & 3.9589 & 0.0104 & BRECHA-C2 & 0.3935 & 0.8127 & BRECHA-C2 & 0.8131 & 0.5288 \\
\hline C2-BRECHA & 0.6610 & 0.6237 & C2-BRECHA & 5.0741 & 0.0012 & C2-BRECHA & 1.5719 & 0.2126 \\
\hline INFLACION-C1 & 0.7420 & 0.5706 & INFLACION-C1 & 0.5815 & 0.6770 & INFLACION-C1 & 0.8804 & 0.4897 \\
\hline C1-INFLACION & 1.3193 & 0.2847 & C1-INFLACION & 2.1854 & 0.0793 & C1-INFLACION & 3.6389 & 0.0181 \\
\hline INFLACION-C2 & 4.2355 & 0.0075 & INFLACION-C2 & 2.3770 & 0.0600 & INFLACION-C2 & 0.7815 & 0.5479 \\
\hline C2-INFLACION & 10.8250 & 0.0000 & C2-INFLACION & 0.3285 & 0.8580 & C2-INFLACION & 1.1734 & 0.3464 \\
\hline PIB-C1 & 1.1262 & 0.3622 & PIB-C1 & 0.7552 & 0.5579 & PIB-C1 & 1.3369 & 0.2839 \\
\hline C1-PIB & 0.2548 & 0.9045 & C1-PIB & 5.2230 & 0.0010 & C1-PIB & 5.8163 & 0.0019 \\
\hline PIB-C2 & 2.4459 & 0.0672 & PIB-C2 & 0.9090 & 0.4635 & PIB-C2 & 0.8601 & 0.5013 \\
\hline C2-PIB & 0.0582 & 0.9934 & C2-PIB & 5.4923 & 0.0007 & C2-PIB & 1.9135 & 0.1395 \\
\hline
\end{tabular}

Fuente: elaboración propia con datos de Banxico. 


\section{Rezagos óptimos del VAR 1978-2017.}

\begin{tabular}{ccccccc}
\hline \multirow{2}{*}{ Rezagos } & LL & LR & FPE & AIC & HQIC & SBIC \\
\hline 0 & 262.85 & & $7.10 \mathrm{E}-11$ & -14.8484 & -14.8024 & -14.7151 \\
1 & 412.09 & 298.480 & $2.40 \mathrm{E}-14$ & -22.8622 & -22.6781 & -22.3290 \\
2 & 438.70 & 53.223 & $8.80 \mathrm{E}-15$ & -23.8686 & -23.5464 & $-22.9354^{*}$ \\
3 & 451.74 & 26.070 & $7.20 \mathrm{E}-15$ & -24.0992 & -23.6389 & -22.7660 \\
4 & 466.45 & $29.432^{*}$ & $5.5 \mathrm{e}-15^{*}$ & $-24.4258^{*}$ & $-23.8275^{*}$ & -22.6927 \\
\hline
\end{tabular}

Fuente: elaboración propia con datos de Banxico.

LL: Log-verosimilitud

LR: Likelihood ratio

FPE: Estadístico del error (Final Prediction Error).

AIC: Criterio de información de Akaike.

HQIC: Criterio de información de Hanna-Quinn.

SBIC: Criterio de información de Schwarz.

***: nivel de significancia al $10 \%$

***: nivel de significancia al $5 \%$

***: nivel de significancia al $1 \%$

\section{Descomposición de varianza.}

\begin{tabular}{|c|c|c|c|c|c|c|}
\hline \multicolumn{7}{|c|}{ Descomposición de la varianza: PIB } \\
\hline Periodo & Error estándar & PIB & INFLACION & BRECHA & C1 & $\mathrm{C} 2$ \\
\hline \multirow[t]{2}{*}{1} & 0.0154 & 100 & 0,0000 & 0.0000 & 0.0000 & 0.0000 \\
\hline & & $(0,0000)$ & $(0.0000)$ & $(0.0000)$ & $(0.0000)$ & $(0.0000)$ \\
\hline \multirow[t]{2}{*}{2} & 0.0160 & 92.81721 & 3.213212 & 1.007402 & 2.942869 & 0.019305 \\
\hline & & $(4,0493)$ & $(2.72)$ & (1.9153) & $(2.6465)$ & $(0.9655)$ \\
\hline \multirow[t]{2}{*}{3} & 0.0161 & 91.93771 & 4.019095 & 1.05264 & 2.90392 & 0.086631 \\
\hline & & $(4,3687)$ & $(3.0727)$ & $(2.106)$ & $(2.6313)$ & (1.3073) \\
\hline \multirow[t]{2}{*}{4} & 0.0171 & 89.93068 & 4.283624 & 2.792818 & 2.915526 & 0.077354 \\
\hline & & $(4.5848)$ & $(2.9392)$ & $(2.7937)$ & $(2.439)$ & $(1.3382)$ \\
\hline \multirow[t]{2}{*}{5} & 0.0189 & 87.66972 & 3.761524 & 2.315443 & 2.444326 & 3.808984 \\
\hline & & $(4.931)$ & $(2.7416)$ & (2.3949) & (2.1916) & (2.7319) \\
\hline \multirow[t]{2}{*}{6} & 0.0194 & 86.82715 & 3.774256 & 2.376519 & 3.378141 & 3.643938 \\
\hline & & $(5.1763)$ & $(2.8579)$ & $(2.4469)$ & $(2.7309)$ & $(2.6977)$ \\
\hline \multirow[t]{2}{*}{7} & 0.0195 & 86.22979 & 4.416794 & 2.355332 & 3.354963 & 3.64312 \\
\hline & & $(5.4556)$ & (3.1962) & $(2.4734)$ & $(2.7953)$ & $(2.6862)$ \\
\hline \multirow[t]{2}{*}{8} & 0.0203 & 85.97481 & 4.304811 & 2.831617 & 3.511419 & 3.37734 \\
\hline & & $(5.5854)$ & (3.1383) & (2.661) & $(2,6759)$ & (2.5723) \\
\hline \multirow[t]{2}{*}{9} & 0.0208 & 86.01843 & 4.496545 & 2.701905 & 3.400706 & 3.382417 \\
\hline & & $(5.6212)$ & $(3.2406)$ & $(2.5821)$ & $(2.6931)$ & (2.6042) \\
\hline \multirow[t]{2}{*}{10} & 0.0212 & 85.77206 & 4.35565 & 2.697794 & 3.610945 & 3.563553 \\
\hline & & $(5.7398)$ & (3.2073) & (2.5656) & (2.8302) & (2.6868) \\
\hline \multirow[t]{2}{*}{11} & 0.0213 & 85.32581 & 4.505913 & 2,74503 & 3.68886 & 3.734387 \\
\hline & & $(5.9517)$ & (3.3494) & $(2.6746)$ & $(2.9415)$ & $(2.7754)$ \\
\hline \multirow[t]{2}{*}{12} & 0.0219 & 85.51805 & 4.399798 & 2.757884 & 3.491041 & 3.833227 \\
\hline & & (5.9873) & (3.2936) & $(2.6981)$ & (2.8382) & (2.8641) \\
\hline
\end{tabular}



http://dx.doi.org/10.22201/fca.24488410e.2018.1204

\begin{tabular}{|c|c|c|c|c|c|c|}
\hline \multicolumn{7}{|c|}{ Descomposicion de la varianza: iNFLACION } \\
\hline Periodo & Error estándar & PIB & INFLACION & BRECHA & $\mathrm{Cl}$ & $\mathrm{C2}$ \\
\hline \multirow[t]{2}{*}{1} & 0.0154 & 100 & 0.0000 & 0.0000 & 0.0000 & 0.0000 \\
\hline & & $(4,3934)$ & (4.3934) & $(0.0000)$ & $(0.0000)$ & $(0.0000)$ \\
\hline \multirow[t]{2}{*}{2} & 0.0160 & 92.81721 & 3.213212 & 1007402 & 2,942869 & 0.019305 \\
\hline & & $(3.4929)$ & $(5.9987)$ & $(0.8409)$ & (1.107) & $(5.1254)$ \\
\hline \multirow[t]{2}{*}{3} & 0.0161 & 91.93771 & 4.019095 & 1.05264 & 2.90392 & 0.086631 \\
\hline & & (3.4473) & (7.0795) & (2.8409) & (1.4357) & $(6.8714)$ \\
\hline \multirow[t]{2}{*}{4} & 0.0171 & 89.93068 & 4.283624 & 2.792818 & 2.915526 & 0.077354 \\
\hline & & $(3.5707)$ & $(7.0396)$ & (3.6621) & $(2.6277)$ & $(7,3256)$ \\
\hline \multirow[t]{2}{*}{5} & 0.0189 & 87.66972 & 3.761524 & 2,315443 & 2.444326 & 3.808984 \\
\hline & & (3.6529) & $(6.7277)$ & (3.6253) & (4.6978) & (7.4491) \\
\hline \multirow[t]{2}{*}{6} & 0.0194 & 86.82715 & 3.774256 & 2.376519 & 3.378141 & 3.643938 \\
\hline & & (3.5967) & $(6.7681)$ & (3.9767) & (5.6595) & (6.9861) \\
\hline \multirow[t]{2}{*}{7} & 0.0195 & 86.22979 & 4.416794 & 2.355332 & 3.354963 & 3.64312 \\
\hline & & (3.9066) & (6.9987) & (4.4903) & (5.9587) & (6.6795) \\
\hline \multirow[t]{2}{*}{8} & 0.0203 & 85.97481 & 4.304811 & 2.831617 & 3.511419 & 3.37734 \\
\hline & & $(4.0802)$ & $(7.1023)$ & $(4.7302)$ & $(6.22)$ & $(6.554)$ \\
\hline \multirow[t]{2}{*}{9} & 0.0208 & 86.01843 & 4.496545 & 2.701905 & 3.400706 & 3.382417 \\
\hline & & (3.9943) & (7.1415) & (4.7599) & $(6.2865)$ & $(6.5909)$ \\
\hline \multirow[t]{2}{*}{10} & 0.0212 & 85.77206 & 4.35565 & 2.697794 & 3.610945 & 3.563553 \\
\hline & & $(4.0769)$ & (7.1614) & (4.771) & (6.2448) & (6.6665) \\
\hline \multirow[t]{2}{*}{ II } & 0.0213 & 85.32581 & 4.505913 & 2,74503 & 3.68886 & 3.734387 \\
\hline & & (4.2604) & (7.1597) & $(4.772)$ & (6.1918) & (6.6688) \\
\hline \multirow[t]{2}{*}{12} & 0.0219 & 85.51805 & 4.399798 & 2.757884 & 3.491041 & 3.833227 \\
\hline & & $(4.4346)$ & (7.1364) & $(4.7512)$ & (6.1368) & $(6,6521)$ \\
\hline
\end{tabular}

\begin{tabular}{|c|c|c|c|c|c|c|}
\hline \multicolumn{7}{|c|}{ Descomposición de la varianza: BRECHA } \\
\hline Periodo & Error estándar & PIB & INFLACION & BRECHA & $\mathrm{Cl}$ & $\mathrm{C}$ \\
\hline \multirow[t]{2}{*}{1} & 0.0154 & 100 & 0.0000 & 0.0000 & 0,0000 & 0,0000 \\
\hline & & (1.1953) & (0.1082) & (1.1828) & $(0.0000)$ & $(0.0000)$ \\
\hline \multirow[t]{2}{*}{2} & 0,0160 & 9281721 & 3.213212 & 1.007402 & 2.942869 & 0.019305 \\
\hline & & $(2.7203)$ & $(1.5545)$ & (1.7829) & $(1.7277)$ & $(0.5207)$ \\
\hline \multirow[t]{2}{*}{3} & 0.0161 & 91.93771 & 4.019095 & 1.05264 & 2.90392 & 0.086631 \\
\hline & & $(4.2147)$ & $(3.186)$ & $(2.0808)$ & $(2.4012)$ & $(0.8709)$ \\
\hline \multirow[t]{2}{*}{4} & 0,0171 & 89,93068 & 4.283624 & 2,792818 & 2.915526 & 0.077354 \\
\hline & & $(5.5153)$ & $(4.3378)$ & $(2.0512)$ & $(3.5093)$ & (1.364) \\
\hline \multirow[t]{2}{*}{5} & 0,0189 & 87.66972 & 3.761524 & 2.315443 & 2.444326 & 3.808984 \\
\hline & & $(5.514)$ & $(4.0748)$ & (1.7833) & (3.4663) & (1.6907) \\
\hline \multirow[t]{2}{*}{6} & 0.0194 & 86.82715 & 3.774256 & 2.376519 & 3.378141 & 3.643938 \\
\hline & & $(6.5216)$ & (4.6061) & (1.7933) & (4.3826) & (2.4523) \\
\hline \multirow[t]{2}{*}{7} & 0.0195 & 86.22979 & 4.416794 & 2,355332 & 3.354963 & 3.64312 \\
\hline & & (7.6247) & (5.5303) & (1.8976) & (5.1106) & (2.9792) \\
\hline \multirow[t]{2}{*}{8} & 0,0203 & 85,97481 & 4,304811 & 2,831617 & 3.511419 & 3.37734 \\
\hline & & $(8.8163)$ & $(6.2828)$ & (2.3051) & $(6.2747)$ & (3.4037) \\
\hline \multirow[t]{2}{*}{9} & 0.0208 & 86,01843 & 4.496545 & 2.701905 & 3.400706 & 3.382417 \\
\hline & & $(9.4192)$ & $(6.4129)$ & $(2.5587)$ & $(6.8507)$ & (3.7873) \\
\hline \multirow[t]{2}{*}{10} & 0.0212 & 85.77206 & 4.35565 & 2.697794 & 3.610945 & 3.563553 \\
\hline & & (10.1579) & $(6.7212)$ & $(2.9234)$ & (7.6755) & (3.9685) \\
\hline \multirow[t]{2}{*}{11} & 0.0213 & 85.32581 & 4.505913 & 2.74503 & 3.68886 & 3.734387 \\
\hline & & (10.7806) & $(7.1559)$ & $(3.2058)$ & $(8.2732)$ & (3.9733) \\
\hline \multirow[t]{2}{*}{12} & 0.0219 & 85.51805 & 4.399798 & 2.757884 & 3.491041 & 3.833227 \\
\hline & & (11.2898) & $(7.4867)$ & $(3.615)$ & (8.8829) & (3.898) \\
\hline
\end{tabular}


E. Modelo VAR 1978-2017.

\begin{tabular}{|c|c|c|c|c|c|}
\hline & PIB & INFLACION & BRECHA & $\mathrm{C} 1$ & $\mathrm{C} 2$ \\
\hline \multirow[t]{3}{*}{$\mathrm{PIB}(-1)$} & 0.13767 & -0.17208 & 0.19695 & -6.58223 & -0.37271 \\
\hline & -0.31552 & -0.45127 & -0.31554 & -6.75748 & -3.17237 \\
\hline & [ 0.43634$]$ & {$[-0.38133]$} & {$[0.62417]$} & {$[-0.97407]$} & {$[-0.11749]$} \\
\hline \multirow[t]{3}{*}{$P I B(-2)$} & -0.04329 & -1.35467 & 0.04875 & -24.28823 & 1.08441 \\
\hline & -0.31309 & -0.44779 & -0.31311 & -6.70542 & -3.14794 \\
\hline & {$[-0.13828]$} & {$[-3.02521]$} & [0.15568] & {$[-3,62218]$} & [ 0.34448$]$ \\
\hline \multirow[t]{3}{*}{$\mathrm{PIB}(-3)$} & -0.08473 & 0.17103 & -0.15007 & 6.88613 & -2.00214 \\
\hline & -0.32475 & -0.46448 & -0.32477 & -6.95528 & -3.26523 \\
\hline & {$[-0.26092]$} & [0.36822] & {$[-0.46207]$} & [0.99006] & {$[-0.61317]$} \\
\hline \multirow[t]{3}{*}{ PIB $(-4)$} & 0.50126 & 0.13564 & 0,50628 & 2.05022 & -0.85435 \\
\hline & $-0,07358$ & -0.10524 & -0.07359 & $-1,57590$ & -0.73983 \\
\hline & [6.81233] & [ 1.28883$]$ & [ 6.88014] & {$[1,30098]$} & [-1.15479] \\
\hline \multirow[t]{3}{*}{ INFLACION $(-1)$} & -0.04979 & 0.64380 & -0.04860 & 0.62636 & -2.11184 \\
\hline & -0.07454 & -0.10661 & -0.07454 & -1.59642 & -0.74946 \\
\hline & {$[-0,66798]$} & {$[6.03884]$} & {$[-0.65197]$} & {$[0,39235]$} & {$[-2.81782]$} \\
\hline \multirow[t]{3}{*}{ INFLACION(-2) } & $-0,03584$ & -0.20371 & $-0,05756$ & 1.96726 & 1.89488 \\
\hline & $-0,08887$ & -0.12711 & -0.08887 & -1.90333 & -0.89354 \\
\hline & {$[-0.40327]$} & {$[-1.60267]$} & {$[-0.64761]$} & [ 1.03359$]$ & [ 2.12065] \\
\hline \multirow[t]{3}{*}{ INFLACION(-3) } & 0.14313 & -0.03269 & 0.13322 & -3.04941 & -1.83195 \\
\hline & -0.08958 & -0.12813 & -0.08959 & $-1,91862$ & -0.90072 \\
\hline & [1.59776] & {$[-0.25514]$} & [1.48704] & {$[-1.58938]$} & {$[-2,03388]$} \\
\hline \multirow[t]{3}{*}{ INFLACION $(-4)$} & $-0,08064$ & 0.14916 & -0.07959 & 1.00072 & -0.04250 \\
\hline & -0.07706 & -0.11022 & -0.07707 & -1.65048 & -0.77484 \\
\hline & {$[-1.04643]$} & [1.35329] & {$[-1.03277]$} & {$[0.60632]$} & {$[-0.05486]$} \\
\hline \multirow[t]{3}{*}{ BRECHA(-1) } & -0.330867 & 0.336214 & 0.607522 & 6.812889 & -0.023477 \\
\hline & -0.31300 & -0.44767 & -0.31302 & -6.70355 & -3.14706 \\
\hline & {$[-1,05708]$} & {$[0.75103]$} & [1.94085] & [ 1.01631$]$ & {$[-0,00746]$} \\
\hline \multirow[t]{3}{*}{$\operatorname{BRECHA}(-2)$} & 0.25056 & 0.91589 & 0.23053 & 16.34444 & -0.57497 \\
\hline & -0.42660 & -0.61015 & -0.42663 & -9.13663 & -4.28929 \\
\hline & [0.58734] & [1.50109] & [0.54036] & [1.78889] & {$[-0.13405]$} \\
\hline \multirow[t]{3}{*}{ BRECHA $(-3)$} & -0.20205 & -1.36385 & -0.03685 & -29.77411 & 2.37016 \\
\hline & -0.42981 & -0.61473 & $-0,42983$ & -9.20521 & $-4,32149$ \\
\hline & {$[-0,47008]$} & {$[-2.21862]$} & {$[-0.08572]$} & {$[-3.23448]$} & [ 0.54846$]$ \\
\hline \multirow[t]{3}{*}{ BRECHA $(-4)$} & 0.122522 & 0,189695 & 0.050219 & 6.927364 & $-1,319529$ \\
\hline & -0.32700 & -0.46769 & -0.32702 & -7.00342 & -3.28783 \\
\hline & [ 0.37468$]$ & {$[0.40560]$} & {$[0.15357]$} & {$[0.98914]$} & {$[-0.40134]$} \\
\hline
\end{tabular}



http://dx.doi.org/10.22201/fca.24488410e.2018.1204

\begin{tabular}{|c|c|c|c|c|c|}
\hline \multirow[t]{3}{*}{$c 1(-1)$} & -0.01067 & 0.01937 & -0.01177 & 1.22243 & -0.02260 \\
\hline & -0.00486 & -0.00695 & -0.00486 & -0.10411 & -0.04887 \\
\hline & {$[-2.19399]$} & [2.78613] & {$[-2,42127]$} & [11.7422] & {$[-0.46235]$} \\
\hline \multirow[t]{3}{*}{$\mathrm{C} 1(-2)$} & 0.01180 & -0.01800 & 0,01405 & $-0,69986$ & 0,12685 \\
\hline & $-0,00691$ & -0.00989 & -0.00691 & -0.14805 & -0.0695 \\
\hline & [1.70741] & [,1.82101] & [ 2.03305] & {$[-4.72735]$} & [1.82517] \\
\hline \multirow[t]{3}{*}{$C 1(-3)$} & -0.009326 & 0.025511 & -0.010117 & 0.608284 & 0.014835 \\
\hline & -0.00696 & -0.00995 & $-0,00696$ & -0.14907 & $-0,06998$ \\
\hline & {$[-1.33993]$} & [2.56267] & {$[-1,45343]$} & [4.08056] & {$[0.21198]$} \\
\hline \multirow[t]{3}{*}{$C 1(-4)$} & 0.00333 & -0.01465 & 0.00381 & -0.20900 & -0.06241 \\
\hline & -0.00476 & -0.00680 & -0.00476 & -0.10187 & -0.04782 \\
\hline & {$[0.70020]$} & {$[-2.15413]$} & [ 0.79985$]$ & {$[-2.05168]$} & {$[-1,30494]$} \\
\hline \multirow[t]{3}{*}{$C 2(-1)$} & 0.00149 & 0.09083 & 0.00064 & 1.87106 & 0.23721 \\
\hline & -0.00856 & -0.01225 & -0.00856 & -0.18337 & -0.08608 \\
\hline & [ 0.17389$]$ & [7.41734] & [0.07482] & [10.2037] & [2.75548] \\
\hline \multirow[t]{3}{*}{$C 2\{-2\}$} & 0.026935 & -0.023032 & 0.029442 & -1.146336 & 0.17131 \\
\hline & -0.01154 & -0.01650 & -0.01154 & -0.24714 & $\cdot 0.11602$ \\
\hline & {$[2,33424]$} & [-1.39557] & [2.55130] & $(-4.63850)$ & [1.47656] \\
\hline \multirow[t]{3}{*}{$C 2(-3)$} & -0.00222 & 0.04677 & -0.00344 & 0.60995 & .0 .21017 \\
\hline & -0.01220 & -0.01745 & -0.01220 & -0.26126 & -0.12265 \\
\hline & {$[-0.18235]$} & [2.68058] & {$[-0.28188]$} & [2.33467] & {$[-1,71357]$} \\
\hline \multirow[t]{3}{*}{$C 2(-4)$} & -0.01749 & -0.03669 & -0.01117 & -1.04962 & -0.31321 \\
\hline & 0,01174 & -0.01679 & -0.01174 & -0.25135 & -0.118 \\
\hline & [-1.49055] & {$[-2.18553]$} & {$[-0.95131]$} & {$[-4.17587]$} & {$[-2.65426]$} \\
\hline \multirow[t]{3}{*}{ c } & 0.024906 & 0.043464 & 0.015692 & 0.36267 & 0.17616 \\
\hline & -0.01133 & -0.01621 & -0.01133 & -0.24266 & $-0,11392$ \\
\hline & [2.19819] & [2.68209] & [1.38484] & [ 1.49454] & [1.54634] \\
\hline \multirow[t]{3}{*}{ D_1989_2008 } & -0.02709 & .0 .01560 & -0.02319 & -0.30286 & -0.06364 \\
\hline & -0.00833 & -0.01192 & -0.00833 & -0.17846 & -0.08378 \\
\hline & {$[-3.25146]$} & [-1,30912] & {$[-2,78263]$} & [-1.69712] & {$[-0.75964]$} \\
\hline \multirow[t]{3}{*}{ D_2009_2017 } & -0.02599 & -0.01703 & -0.02098 & -0.36589 & -0.08052 \\
\hline & -0.00895 & -0.0128 & -0.00895 & -0.19173 & -0.09001 \\
\hline & {$[-2.90256]$} & {$[-1.33030]$} & {$[-2,34369]$} & (-1.90833) & {$[-0.89458]$} \\
\hline R-squared & 0,5796 & 0.9100 & 0.9438 & 0,9691 & 0.3692 \\
\hline Adj. R-squared & 0,5096 & 0.8950 & 0,9344 & 0,9640 & 0.2641 \\
\hline F-statistic & 8.2727 & 60.6574 & 100.7458 & 188.3312 & 3.5124 \\
\hline Log likelihood & 439.4375 & 383.9712 & 439.4286 & -35.5120 & 81.6943 \\
\hline Akaike AIC & -5.3734 & -4.6577 & -5.3733 & 0.7550 & -0.7573 \\
\hline Schwarz SC & -4.9218 & -4.2061 & -4.9217 & 1.2066 & -0.3057 \\
\hline
\end{tabular}



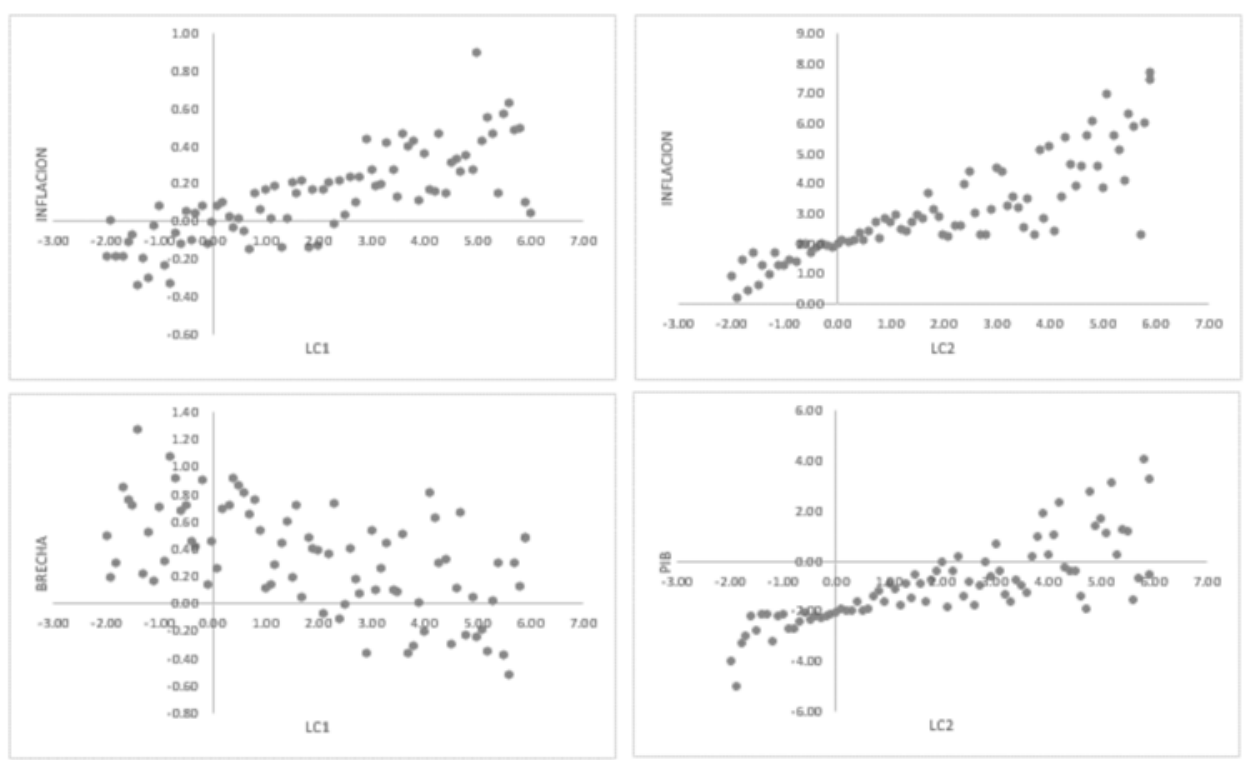

Figura 4a

Diagramas de dispersión (1978-1988).

Fuente: elaboración propia con datos de Banxico.
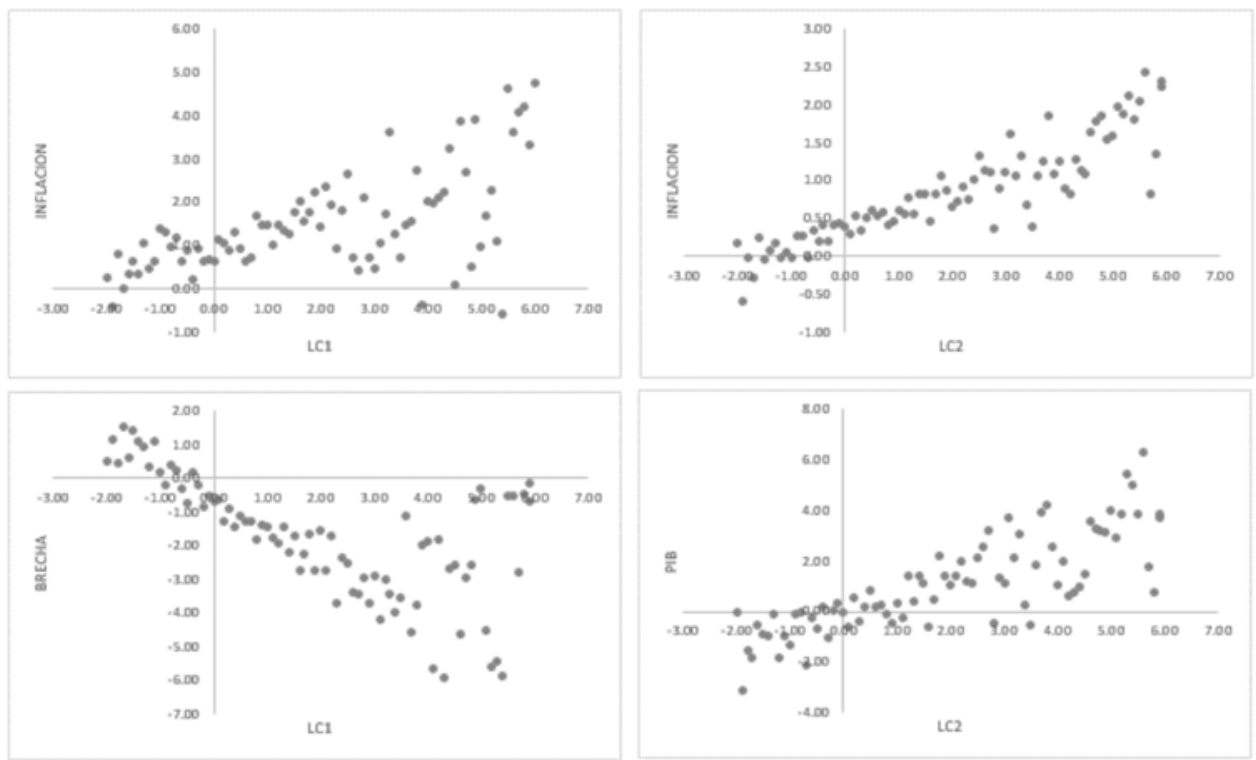

Figura 4b

Diagramas de dispersión (1989-2008).

Fuente: elaboración propia con datos de Banxico. 

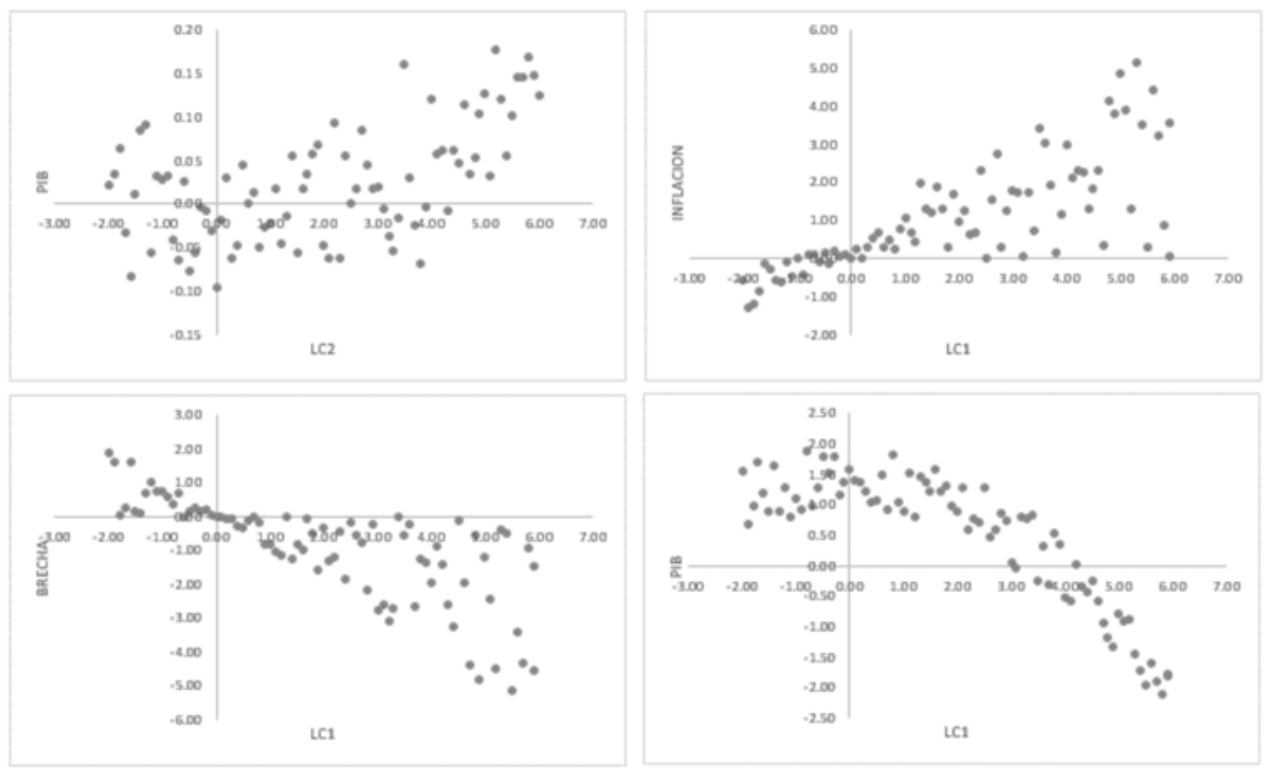

Figura 4c

Diagramas de dispersión (2009-2017).

Fuente: elaboración propia con datos de Banxico. 